\title{
Sequences of enumerative geometry: congruences and asymptotics
}

\author{
Daniel B. Grünberg, Pieter Moree \\ Appendix by Don Zagier
}

\begin{abstract}
We study the integer sequence $v_{n}$ of numbers of lines in hypersurfaces of degree $2 n-3$ of $\mathbb{P}^{n}, n>1$. We prove a number of congruence properties of these numbers of several different types. Furthermore, the asymptotics of the $v_{n}$ are described (in an appendix by Don Zagier). An attempt is made at a similar analysis of two other enumerative sequences: the numbers of rational plane curves and the numbers of instantons in the quintic threefold.
\end{abstract}

We study the sequence of numbers of lines in a hypersurface of degree $D=2 n-3$ of $\mathbb{P}^{n}, n>1$. The sequence is defined by (see e.g. [8])

$$
v_{n}:=\int_{G(2, n+1)} c_{2 n-2}\left(\operatorname{Sym}^{D} Q\right),
$$

where $G(2, n+1)$ is the Grassmannian of $\mathbb{C}^{2}$ subspaces of $\mathbb{C}^{n+1}$ (i.e. projective lines in $\left.\mathbb{P}^{n}\right)$ of dimension $2(n+1-2)=2 n-2, Q$ is the bundle of linear forms on the line (of rank $r=2$, corresponding to a particular point of the Grassmannian), and $\operatorname{Sym}^{D}$ is its $D$ th symmetric product - of rank $\left(\begin{array}{c}D+r-1 \\ r-1\end{array}\right)=D-1=2 n-2$. The top Chern class (Euler class) $c_{2 n-2}$ is the class dual to the 0-chain (i.e. points) corresponding to the zeros of the bundle $\operatorname{Sym}^{D}(Q)$, i.e. to the vanishing of a degree $D$ equation in $\mathbb{P}^{n}$; this is the geometric requirement that the lines lie in a hypersurface.

The integral (11) can actually be written as a sum:

$$
v_{n}=\sum_{0 \leq i<j \leq n} \frac{\prod_{a=0}^{D}\left(a w_{i}+(D-a) w_{j}\right)}{\prod_{0 \leq k \leq n, k \neq i, j}\left(w_{i}-w_{k}\right)\left(w_{j}-w_{k}\right)},
$$

where $w_{0}, \ldots, w_{n}$ are arbitrary complex variables. This is a consequence of a localisation formula due to Atiyah and Bott from equivariant cohomology, which says that only the (isolated) fixed points of the $\left(\mathbb{C}^{*}\right)^{n+1}$ action contribute to the defining integral of $v_{n}$. Hence the sum. For the first few values of $n$ computation yields $v_{2}=1, v_{3}=27$, $v_{4}=2875, v_{5}=698005, v_{6}=305093061$.

D. Zagier gave a simple proof that the right hand side of (2) is independent of Mathematics Subject Classification (2000). 14N10, 11A07, 41A60 
$w_{0}, \ldots, w_{n}$ (as it must be for (2) to hold), and that in fact it can be replaced by the much simpler formula

$$
v_{n}=\left[(1-x) \prod_{j=0}^{2 n-3}(2 n-3-j+j x)\right]_{x^{n-1}}
$$

where the notation $[\ldots]_{x^{n}}$ means the coefficient of $x^{n}$. In fact, formula (2) was proved in a very different way using methods from Schubert calculus by B.L. van der Waerden, who established it in part 2 of his celebrated 20 part 'Zur algebraischen Geometrie' series of papers [19, 20]. The number of linear subspaces of dimension $k$ contained in a generic hypersurface of degree $d$ in $\mathbb{P}^{n}$, when it is finite, can be likewise expressed as the coefficient of a monomial in a certain polynomial in several variables, see e.g. [15. Theorem 3.5.18].

Zagier also gave the formula

$$
v_{n} \sim \sqrt{\frac{27}{\pi}}(2 n-3)^{2 n-7 / 2}\left(1-\frac{9}{8 n}-\frac{111}{640 n^{2}}-\frac{9999}{25600 n^{3}}+\cdots\right),
$$

where the right hand side is an asymptotic expansion in powers of $n^{-1}$ with rational coefficients that can be explicitly computed. The proof of this formula, as well as the derivation of (3) from (2), can be found in the appendix.

The remaining results, summarized in Theorem 1 and Theorem 2, are concerned with congruence properties of the numbers $v_{n}$. In this context it turns out to be convenient to define $v_{1}=1$ (even though there is no such thing as a hypersurface in $\mathbb{P}^{1}$ of degree -1 ) and even more remarkably $v_{0}=-1$. We do not doubt that the congruence results presented here form only the tip of an iceberg.

A first version of this paper was single authored by the first author. Indeed, the present version of this paper is similar to the first one, except for sections 2 and 3 which have been greatly revised and expanded by the second author. The conjectures outside these two sections are due to the first author alone. Sections 4 and 5 were revised by both the second author and Don Zagier.

\section{Introduction}

The motivating idea behind this paper is the expectation that certain problems in enumerative geometry are coupled to modularity. This is a recurrent theme in string theory, where partition functions have often an enumerative interpretation as counting objects (instantons, etc) and must satisfy the condition of modularity covariance in order to obtain the same amplitude when two worldsheets have the same intrinsic geometry.

Modular forms, as is well known, have Fourier coefficients satisfying many interesting congruences (think of Ramanujan's congruences for partitions, or for his function $\tau(n))$. The same can happen for the coefficients of expansions related to modular forms, e.g. the expansions $y=\sum A_{n} x^{n}$ obtained by writing a modular form $y$ (locally) as a power series in a modular function $x$. For instance, the famous Apéry numbers related to Apéry's proof of the irrationality of $\zeta(3)$ are obtainable in this way 3 and satisfy many interesting congruences [18]. The numbers appearing in the context of mirror symmetry, Picard-Fuchs equations for Calabi-Yau manifolds, Gromov-Witten invariants 
and similar problems of enumerative geometry are sometimes related to modular forms and sometimes not, so we can reasonably hope for interesting congruence properties in these contexts also. In Section 2 we shall find astonishingly many congruences for our sequence $v_{n}$. We shall first draw a few tables for congruences mod 2,3,4,5 or 11, and then summarize the observed congruences. In Section 3 we prove those congruences by elementary means starting from (3), and a few conjectures will be formulated. Sequences of numbers coming from modular forms also often have interesting asymptotic properties and we therefore wish to study this, too. In Section 4 we find the asymptotic properties of the $v_{n}$ numerically by using a clever empirical trick shown to us by Don Zagier which we call the $\operatorname{asymp}_{k}$ trick. (A rigorous proof of these asymptotics, as already mentioned above, was also provided by him and is reproduced in the appendix.) Section 5 presents congruences and asymptotics for two further examples of enumerative sequences, without proofs. The sequence of rational curves on the plane and the sequence of instantons on the quintic threefolds partly partly mimic the behaviour of the original sequence of lines in hypersurfaces.

\section{Congruences}

We will consider the sequences $\left\{v_{n}(\bmod k)\right\}_{k=1}^{\infty}$ for some small values of $k$; that is, we study the reduction of the integers $v_{n}$ modulo $k$. It turns out to be instructive to order the $v_{n}$ modulo $k$ in a table. Each table has $k$ columns. The $i$ th column $(i=1, \ldots, k)$ gives the values of $v_{l k+i}(\bmod k)(l \geq 0)$.

For instance, the first few tables at $k=2,3,4, \ldots$ look like

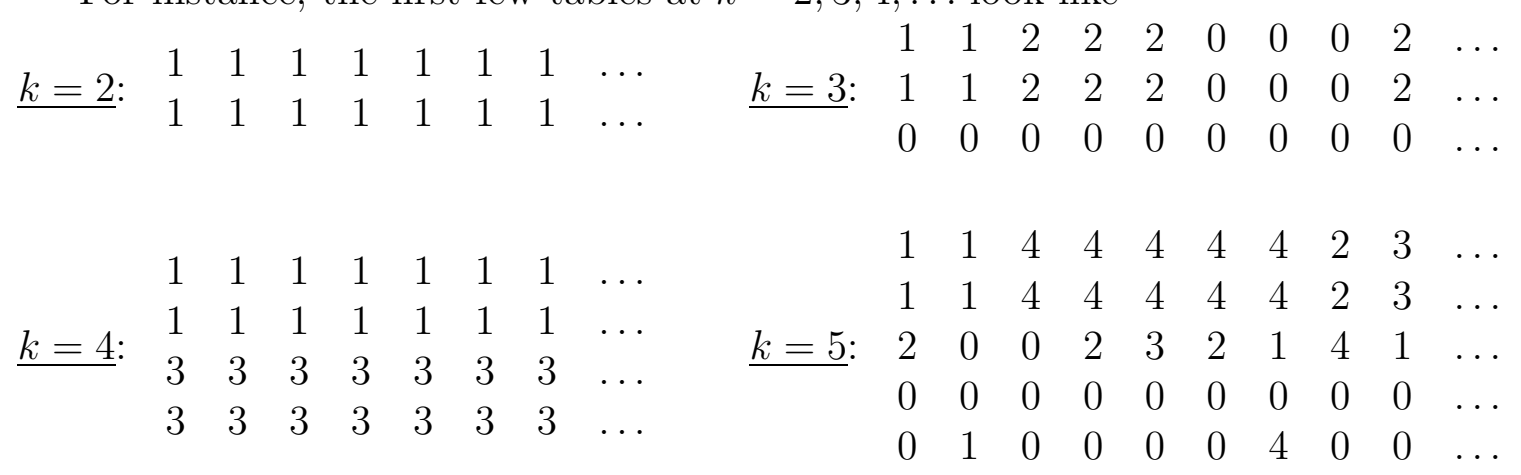

The first table $(k=2)$ says that all the $v_{n}$ are odd integers. We shall mostly be interested in the tables for prime $k$. Here is a typical prime table:

$\begin{array}{cccccccccccccccl}1 & 1 & 10 & 10 & 10 & 10 & 10 & 10 & 10 & 10 & 10 & 10 & 10 & 8 & \ldots \\ 1 & 1 & 10 & 10 & 10 & 10 & 10 & 10 & 10 & 10 & 10 & 10 & 10 & 8 & \ldots \\ 5 & 9 & 10 & 7 & 8 & 6 & 10 & 2 & 7 & 8 & 6 & 10 & 8 & 5 & \ldots \\ 4 & 1 & 5 & 8 & 6 & 7 & 10 & 8 & 2 & 6 & 7 & 10 & 8 & 8 & \ldots \\ & 0 & 9 & 3 & 2 & 2 & 0 & 0 & 0 & 0 & 0 & 0 & 0 & 1 & 5 & \ldots \\ & 9 & 3 & 10 & 0 & 1 & 4 & 8 & 10 & 7 & 6 & 2 & 8 & 10 & 7 & \ldots \\ 0 & 0 & 0 & 0 & 0 & 0 & 0 & 0 & 0 & 0 & 0 & 0 & 0 & 0 & \ldots \\ 0 & 2 & 5 & 1 & 1 & 0 & 0 & 0 & 0 & 0 & 0 & 0 & 8 & 10 & \ldots \\ 0 & 2 & 2 & 2 & 7 & 0 & 0 & 0 & 0 & 0 & 0 & 0 & 10 & 1 & \ldots \\ 0 & 10 & 0 & 10 & 2 & 0 & 0 & 0 & 0 & 0 & 0 & 0 & 8 & 3 & \ldots \\ 0 & 2 & 8 & 9 & 3 & 0 & 0 & 0 & 0 & 0 & 0 & 0 & 7 & 5 & \ldots\end{array}$

Study of these and other tables led us to formulate a number of conjectures, most of 
which we were able to prove. An overview of these results is given in Theorem 1.

Theorem 1 The following holds for the tables of $v_{n} \bmod k$ :

1. All $v_{n}$ are odd.

2. The first two rows of each table are equal.

3. If $k$ is even, then rows $k / 2+1$ and $k / 2+2$ are equal.

4. For $k$ odd, row $(k+3) / 2$ contains only zeros.

5. For $k$ prime, the first two rows start with 1,1 followed by $k$ occurrences of $k-1$.

6. For $k>2$ prime, the last $(k-1) / 2$ slots of the first column vanish.

7. For $k>2$ prime, there is a block of zeros at the bottom (after $(k-1) / 2$ columns), of height $(k-1) / 2$ and width $(k+3) / 2$.

8. For $k=2^{q}$, all rows are constant and in two fold way sweep out all odd residues, i.e. for every odd integer a with $1 \leq a \leq 2^{q}$ there are precisly two rows that have only a as entry.

9. For $k=2^{q}>2$ the entries in the rows $1,2,2^{q-1}, 2^{q-1}+1,2^{q-1}+2,2^{q}-1$ equal, respectively, $1,1,2^{q-1}-1,2^{q-1}+1,2^{q-1}+1,2^{q}-1$.

10. For $k=2^{q}>2$ the entries in row $a$ and row $a+2^{q-1}$ differ by $2^{q-1}\left(\bmod 2^{q}\right)$.

Proof. These ten claims are proved in, respectively, lemmas 6, 3, 3, 4, 7, \& 8, 11, 13, 20] \& 23, 22 and 22

On computing the reductions of $v_{1}, \ldots, v_{32}$ modulo 32 one finds by part 8 of this theorem that for $k=4$ the table has constant rows 1,1,3,3, for $k=8$ constant rows $1,1,3,3,5,5,7,7$, for $k=16$ constant rows $1,1,11,11,5,5,7,7,9,9,3,3,13,13$, 15,15 and for $k=32$ constant rows $1,1,27,27,21,5,7,23,9,9,19,19,29,13,31,15,17,17$, $11,11,5,21,23,7,25,25,3,3,13,29,15,31$. Thus, for modulus $2^{q}$ with $q \leq 3$ we observe that pairs of values occur and that these, moreover, are in ascending order. For $q=4$ the values still come in pairs, but the order is no longer ascending. For $n \geq 5$ it turns out that pairs with equal values become sparser and sparser. Notice that in the above cases for every modulus all odd values are assumed exactly twice. By part 8 this always happens. Thus, given an odd integer $a$ and any integer $q \geq 1$ there are infinitely many integers $m$ such that $v_{m} \equiv a\left(\bmod 2^{q}\right)$ (or put more succinctly: modulo powers of two the sequence $v_{n}$ is equidistributed over the odd residue classes).

For $k$ prime, often $v_{n} \equiv 0(\bmod k)$ for trivial reasons. It then makes sense to consider divisibility of $v_{n}$ by higher powers of $k$. Our deepest result in this direction is provided by the following theorem.

Theorem 2 1. If $p \geq 5$ is a prime, then

$$
v_{\frac{p+3}{2}} \equiv-2 p^{3}\left(\bmod p^{4}\right) \text { and } v_{\frac{p+3}{2}} \equiv 2 p^{3}(1-p)(p-1) ! 4^{p-1}\left(\bmod p^{5}\right) \text {. }
$$

2. Let $r \geq 1$ and $p \geq 2 r+1$ be a prime. Then

$$
v_{\frac{p+3}{2}+r p} \equiv C_{r} p^{2 r+2}\left(\bmod p^{2 r+3}\right),
$$

where

$$
C_{r}=\frac{r}{(-4)^{r-1}}\left(\frac{2 r+1}{r !}\right)^{2} \sum_{j=0}^{2 r} b_{j, r}((1-2 j))_{2 r-1},
$$

the integers $b_{j, r}$ are defined implicitly by $\prod_{a=1}^{2 r}(2 r+1-a+a x)=\sum_{j=0}^{2 r} b_{j, r} x^{j}$, and $((u))_{a}:=\prod_{j=1}^{a}(u+2 j-2)$. 
Remark. Note that $b_{2 r-j, r}=b_{j, r}$. Numerical experimentation suggests that the numerator of $c_{r}$ always equals a power of 2 and that the congruence (5) holds for all odd primes.

Below we record some values of $c_{r}$.

$$
\begin{array}{cccccc} 
& 1 & 2 & 3 & 4 & 5 \\
c_{r}: & -81 & \frac{103125}{8} & -\frac{210171535}{64} & \frac{1308348857025}{1024} & -\frac{11660783598520749}{16384}
\end{array}
$$

\section{Proofs of the theorems}

\subsection{Some generalities}

First recall from the elementary theory of finite fields of order $p$, that

$$
x^{p-1}-1 \equiv \prod_{j=1}^{p-1}(x-j)(\bmod p) .
$$

(Here and below the letter $x$ denotes a variable.) By substituting $x=0$ one obtains Wilson's theorem:

$$
(p-1) ! \equiv-1(\bmod p) .
$$

We also recall the freshman's identity $(a+b)^{p} \equiv a^{p}+b^{p}(\bmod p)$, from which we infer that if $f(x) \in \mathbb{Z}[x]$, then $f(x)^{p} \equiv f\left(x^{p}\right)(\bmod p)$. These results will be freely used in the sequel, without further referring to them.

Lemma 1 We have $v_{n} \equiv 0\left(\bmod (2 n-3)^{2}\right)$.

Proof. The term with $j=0$ in (3) equals $2 n-3$. The term with $j=2 n-3$ equals $(2 n-3) x$. Hence $v_{n}=(2 n-3)^{2} w_{n}$, where $w_{n}=\left[(1-x) \prod_{j=1}^{2 n-4}(2 n-3-j+j x)\right]_{x^{n-2}}$.

The following result was first noticed by D. Kerner. An alternative, slightly longer, proof was given by M. Vlasenko.

Lemma 2 We have $v_{n} \equiv 0\left(\bmod (2 n-3)^{3}\right)$.

Proof. It suffices to show that $w_{n} \equiv 0(\bmod 2 n-3)$. Note that, modulo $2 n-3$, we have that

$$
\begin{aligned}
w_{n} & \equiv\left[(1-x) \prod_{j=1}^{2 n-4}(-j+j x)\right]_{x^{n-2}} \equiv-(2 n-4) !\left[(x-1)^{2 n-3}\right]_{x^{n-2}} \\
& =-(2 n-4) !\left(\begin{array}{c}
2 n-3 \\
n-2
\end{array}\right)=-(2 n-4) ! \frac{2 n-3}{n-1}\left(\begin{array}{c}
2 n-4 \\
n-2
\end{array}\right)=-(2 n-3) ! C_{n-2},
\end{aligned}
$$

where $C_{m}:=\frac{1}{m+1}\left(\begin{array}{c}2 m \\ m\end{array}\right)$ is the $m$ th Catalan number. The Catalan numbers are integers that arise in numerous counting problems.

Remark. It might be interesting to see whether the integers $v_{n} /(2 n-3)^{i}$ with $i=1,2$ or 3 also have a geometric meaning.

The next result was obtained in collaboration with Alexander Blessing. It establisheds parts 2 and 3 of Theorem 11. 
Lemma 3 For $l \geq 0$ we have $v_{l n+1} \equiv v_{l n+2}(\bmod 2 n)$.

Proof. Since $v_{1}=v_{2}=1$ the result is trivially true for $l=0$ and thus we may assume $l \geq 1$. We have, modulo $2 n$,

$$
v_{l n+1} \equiv\left[(1-x) \prod_{j=0}^{2 l n-1}(-1-j+j x)\right]_{x^{l n}} .
$$

Furthermore, we have, modulo $2 n$,

$$
\begin{aligned}
v_{l n+2} & \equiv\left[(1-x) \prod_{j=0}^{2 l n+1}(1-j+j x)\right]_{x^{l n+1}} \equiv\left[(1-x) x \prod_{j=0}^{2 l n}(1-j+j x)\right]_{x^{l n+1}} \\
& \equiv\left[(1-x) \prod_{j=0}^{2 l n}(1-j+j x)\right]_{x^{l n}} \equiv\left[(1-x) \prod_{j=0}^{2 l n}(1-(2 l n-j)+(2 l n-j) x)\right]_{x^{l n}} \\
& \equiv\left[(1-x) \prod_{j=0}^{2 l n}(1+j-j x)\right]_{x^{l n}} \equiv\left[(1-x) \prod_{j=0}^{2 l n-1}(-1)(-1-j+j x)\right]_{x^{l n}} \\
& \equiv\left[(1-x) \prod_{j=0}^{2 l n-1}(-1-j+j x)\right]_{x^{l n}} \equiv v_{l n+1} .
\end{aligned}
$$

This concludes the proof.

The next lemma generalizes Lemma 1. It implies part 4 of Theorem 1.

Lemma 4 If $k$ is odd, then $v_{l k+(k+3) / 2} \equiv 0\left(\bmod (2 l+1)^{2} k^{2 l+2}\right)$.

Proof. We have $v_{l k+(k+3) / 2} \equiv\left[(1-x) \prod_{j=0}^{(2 l+1) k}((2 l+1) k-j+j x)\right]_{x^{l k+(k+1) / 2}}$. The terms in the product with $j=0$ and $j=(2 l+1) k$ lead to a factor of $(2 l+1)^{2} k^{2}$. The remaining terms in the product that are divisible by $k$ lead to a factor $k^{2 l}$.

\subsection{The sequence $\left\{v_{n}\right\}_{n=1}^{\infty}$ modulo primes}

The following lemma will be repeatedly used in this section.

Lemma 5 Let $p$ be a prime and $c$ an integer. Then, modulo $p$,

$$
\prod_{i=1}^{p-1}(i x-i+c) \equiv \begin{cases}-(x-1)^{p-1} & \text { if } p \mid c, \\ -\left(x+x^{2}+\ldots+x^{p-1}\right)=\frac{x^{p}-x}{1-x} & \text { otherwise. }\end{cases}
$$

Proof. If $p \mid c$, then the result is trivial, so assume $p \nmid c$. We can write

$$
\prod_{i=1}^{p-1}(i x-i+c) \equiv \prod_{i=1}^{p-1} i \prod_{i=1}^{p-1}(x-1+c / i) \equiv \frac{x^{p}-x}{1-x},
$$

where we have used that as $i$ runs over $1,2, \cdots, p-1,-1+c / i$ runs over all residues modulo $p$, except for -1 .

The second lemma in this section is part 1 of Theorem 1 . 
Lemma 6 For $n \geq 1$ we have $v_{n} \equiv 1(\bmod 2)$.

Proof. Modulo 2, the $2 n-2$ terms in the product in (3) are alternatingly 1 and $x$. It thus follows that $v_{n} \equiv\left[(1-x) x^{n-1}\right]_{x^{n-1}} \equiv 1(\bmod 2)$. This concludes the proof.

The next lemma together with Lemma 8 establishes part 5 of Theorem 1 .

Lemma 7 Let $p$ be a prime. Then $v_{p+1} \equiv v_{p+2} \equiv 1(\bmod p)$.

Proof. Modulo $p$ we have $v_{p+1} \equiv\left[(1-x) \prod_{j=1}^{p-1}(-1-j-j x)^{2}\right]_{x^{p}} \equiv\left[(1-x)\left(\frac{x^{p}-x}{1-x}\right)^{2}\right]_{x^{p}}$ where in the derivation of the first congruence we noted that modulo $p$ the $j$ th term in the product (3) is equal to the $(j+p)$ th and in the second we used lemma 5 . Now note that

$$
\left[(1-x)\left(\frac{x^{p}-x}{1-x}\right)^{2}\right]_{x^{p}}=\left[(1-x)\left(\frac{x}{1-x}\right)^{2}\right]_{x^{p}}=\left[\sum_{k \geq 2} x^{k}\right]_{x^{p}}=1 .
$$

Finally, by Lemma 3, $v_{p+2}$ satisfies the same congruence as $v_{p+1}$ modulo $p$.

The proof of the next lemma involves congruences for binomial coefficients. In all cases these can be found by direct computation, but often it is more convenient to invoke a classical result of E. Lucas. Let $n \geq m$ be natural numbers and write $n=$ $a_{0}+a_{1} p+a_{2} p^{2}+\cdots+a_{s} p^{s}$ and $m=b_{0}+b_{1} p+b_{2} p^{2}+\cdots+b_{s} p^{s}$ with $0 \leq a_{i}, b_{i} \leq p-1$. Then Lucas's theorem states that

$$
\left(\begin{array}{l}
n \\
m
\end{array}\right) \equiv\left(\begin{array}{l}
a_{0} \\
b_{0}
\end{array}\right)\left(\begin{array}{l}
a_{1} \\
b_{1}
\end{array}\right) \cdots\left(\begin{array}{l}
a_{s} \\
b_{s}
\end{array}\right)(\bmod p)
$$

Recall that $\left(\begin{array}{l}a \\ b\end{array}\right)=0$ if $b>a$. For example, by direct computation we find that

$$
\left(\begin{array}{l}
p^{2}-2 \\
2 p-2
\end{array}\right) \equiv\left[\frac{(-2) \cdots(-2 p+1)}{1 \cdots(2 p-2)}\right]^{\prime}(1-p) \equiv-(2 p-1)(p-1) \equiv-1(\bmod p)
$$

$\left([\ldots]^{\prime}\right.$ means skipping multiples of $\left.p\right)$. By Lucas's theorem we find that

$$
\left(\begin{array}{l}
p^{2}-2 \\
2 p-2
\end{array}\right)=\left(\begin{array}{c}
(p-1) p+p-2 \\
1 \cdot p+p-2
\end{array}\right) \equiv\left(\begin{array}{c}
p-1 \\
1
\end{array}\right)\left(\begin{array}{l}
p-2 \\
p-2
\end{array}\right) \equiv-1(\bmod p)
$$

Likewise we immediately find using Lucas' theorem that, with $r=1$ and $p>3$,

$$
\left(\begin{array}{c}
2 p-1 \\
p-1
\end{array}\right) \equiv 1\left(\bmod p^{r}\right)
$$

(This identity with $r=2$ was proved in 1819 by Charles Babbage. For $r=3$ it follows from Wolstenholme's theorem, see F.L. Bauer [1].)

At various points we use the easy result that $\left(\begin{array}{c}p-1 \\ j\end{array}\right) \equiv(-1)^{j}(\bmod p)$. To see this observe that modulo $p$ the entries, except the two outmost ones, in the $(p+1)$ th row of Pascal's triangle are zero modulo $p$. Since each of these entries arises as sum of two elements above it in the $p$ th row, the entries in the $p$ th alternate between 1 and -1 . Similarly one infers that $\left(\begin{array}{c}p-2 \\ j\end{array}\right) \equiv(-1)^{j}(j+1)(\bmod p)$.

For a nice survey of arithmetic properties of binomial coefficients we refer the reader to Granville [9]. 
Lemma 8 Let $p$ be a prime and $2 \leq l \leq p+1$. Then $v_{l p+1} \equiv v_{l p+2} \equiv-1(\bmod p)$.

Proof. We have $v_{l p+1} \equiv\left[(1-x) P_{p}^{l}(x)\right]_{x^{l p}}(\bmod p)$, say, with $P_{p}^{l}(x):=\prod_{i=1}^{p-1}(i x-i-1)^{2 l}$. As before

$$
\begin{aligned}
& P_{p}^{l}(x) \equiv\left(x+x^{2}+\ldots+x^{p-1}\right)^{2 l} \equiv x^{2 l}\left[\left(1-x^{p-1}\right)\left(1+x+x^{2}+\ldots\right)\right]^{2 l}(\bmod p)
\end{aligned}
$$

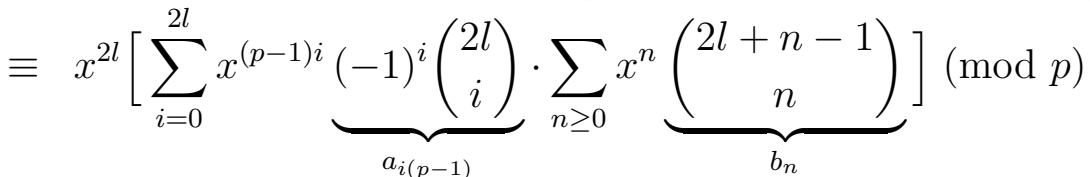

$$
\begin{aligned}
& \equiv x^{2 l}\left[\ldots+x^{l(p-2)-1} \sum_{i=0}^{l-1} a_{(l-1-i)(p-1)} b_{(i+1)(p-1)-l-1}\right. \\
& \left.+x^{l(p-2)} \sum_{i=0}^{l-1} a_{(l-1-i)(p-1)} b_{(i+1)(p-1)-l}+\ldots\right](\bmod p),
\end{aligned}
$$

where we have used that

$$
\left(\sum_{k \geq 0} x^{k}\right)^{2 l}=\sum_{n \geq 0}\left(\begin{array}{c}
2 l+n-1 \\
n
\end{array}\right) x^{n}
$$

Thus

$$
\begin{aligned}
v_{l p+1} & \equiv \sum_{i=0}^{l-1} a_{(l-1-i)(p-1)}\left(b_{(i+1)(p-1)-l}-b_{(i+1)(p-1)-l-1}\right)(\bmod p) \\
& \equiv \sum_{i=0}^{l-1} a_{(l-1-i)(p-1)}\left(\begin{array}{c}
(i+1)(p-1)+l-2 \\
2 l-2
\end{array}\right)(\bmod p) .
\end{aligned}
$$

Note that

$$
a_{(l-1-i)(p-1)}=(-1)^{l-1-i}\left(\begin{array}{c}
2 l \\
l-1-i
\end{array}\right)= \begin{cases}-2 l & \text { for } i=l-2 \\
1 & \text { for } i=l-1\end{cases}
$$

and, furthermore,

$$
a_{(l-1-i)(p-1)}=\left\{\begin{aligned}
0(\bmod p) & \text { for } i=0, \ldots, p-l-2 \\
-1(\bmod p) & \text { for } i=p-l-1
\end{aligned}\right.
$$

The remainder of the proof requires a separate discussion for $l \leq(p-1) / 2$, for $l=$ $(p+1) / 2,(p+1) / 2<l<p$, for $l=p$ and for $l=p+1$.

The case $l \leq(p-1) / 2$ :

Note that

$$
\begin{aligned}
&\left(\begin{array}{c}
(i+1)(p-1)+l-2 \\
2 l-2
\end{array}\right)= \frac{[(i+1) p-i-l] \cdots[(i+1) p-i+l-3]}{1 \cdots(2 l-2)} \\
& \equiv \begin{cases}0(\bmod p) & \text { for } i=0, \ldots, l-3 ; \\
\frac{(-1) \cdots(-2 l+2)}{1 \cdots(2 l-2)} \equiv 1(\bmod p) & \text { for } i=l-2 \\
\frac{(-2) \cdots(-2 l+1)}{1 \cdots(2 l-2)} \equiv 2 l-1(\bmod p) & \text { for } i=l-1 .\end{cases}
\end{aligned}
$$


Hence in the sum for $v_{l p+1}$, only the terms $i=l-2$ and $i=l-1$ contribute, that is: $v_{l p+1}=-2 l \cdot 1+1 \cdot(2 l-1) \equiv-1(\bmod p)$.

The case $l=(p+1) / 2$ :

Here we find that

$$
\left(\begin{array}{cl}
(i+1)(p-1)+l-2 \\
2 l-2
\end{array}\right) \equiv \begin{cases}1(\bmod p) & \text { for } i=l-2 \\
0(\bmod p) & \text { for } 0 \leq i \leq l-1, i \neq l-2\end{cases}
$$

Hence in the sum for $v_{l p+1}$, only the term $i=l-2$ contributes and we infer that $v_{l p+1} \equiv-2 l \cdot 1 \equiv-1(\bmod p)$.

The case $(p+1) / 2<l<p$ :

Here we compute that

$$
\left(\begin{array}{cl}
(i+1)(p-1)+l-2 \\
2 l-2
\end{array}\right) \equiv \begin{cases}0(\bmod p) & \text { for } i=p-l, \ldots, l-3 \\
-l(\bmod p) & \text { for } i=p-l-1 \\
2-l(\bmod p) & \text { for } i=l-2 \\
(1-2 l)(l-1)(\bmod p) & \text { for } i=l-1\end{cases}
$$

Hence in the sum for $v_{l p+1}$, only the terms $i=p-l-1, i=l-2$ and $i=l-1$ contribute. That is: $v_{l p+1} \equiv-1 \cdot(-l)-2 l \cdot(2-l)+1 \cdot(1-2 l)(l-1) \equiv-1(\bmod p)$.

The case $l=p$ :

Note that

$$
a_{(p-1-i)(p-1)}=(-1)^{p-1-i}\left(\begin{array}{c}
2 p \\
p-1-i
\end{array}\right)= \begin{cases}0(\bmod p) & \text { for } i=0, \ldots, p-2 \\
1(\bmod p) & \text { for } i=p-1\end{cases}
$$

while for $i=p-1,\left(\begin{array}{c}(i+1)(p-1)+p-2 \\ 2 p-2\end{array}\right)$ boils down to $\left(\begin{array}{c}p^{2}-2 \\ 2 p-2\end{array}\right) \equiv-1(\bmod p)$ (see the preamble to this lemma). Hence $v_{l p+1} \equiv-1(\bmod p)$.

The case $l=p+1$ :

Here we find that

$$
a_{(p-i)(p-1)}=(-1)^{i+1}\left(\begin{array}{c}
2 p+2 \\
p-i
\end{array}\right) \equiv\left\{\begin{aligned}
-2(\bmod p) & \text { for } i=0 \\
0(\bmod p) & \text { for } i=1, \ldots, l-4 \\
1(\bmod p) & \text { for } i=l-3 \\
-2(\bmod p) & \text { for } i=l-2 \\
1(\bmod p) & \text { for } i=l-1
\end{aligned}\right.
$$

and

$$
\left(\begin{array}{cl}
(i+2)(p-1) \\
2 p
\end{array}\right) \equiv \begin{cases}0(\bmod p) & \text { for } i=0 \\
1(\bmod p) & \text { for } i=l-3 \\
1(\bmod p) & \text { for } i=l-2 \\
0(\bmod p) & \text { for } i=l-1\end{cases}
$$

Hence $v_{l p+1} \equiv-2 \cdot 0+1 \cdot 1-2 \cdot 1+1 \cdot 0 \equiv-1(\bmod p)$.

This proves that in all cases, $v_{l p+1} \equiv-1(\bmod p)$. By lemma 3 , the same is true for $v_{l p+2}$.

The cases $l=p$ and $l=p+1$ in the latter proof can be proved more succinctly as is done in the proofs of Lemma 9, respectively Lemma 10.

Lemma 9 Let $p$ be a prime. Then $v_{p^{2}+1} \equiv v_{p^{2}+2} \equiv-1(\bmod p)$. 
Proof. Note that, modulo $p$, the integer $v_{p^{2}+1}$ is congruent to

$$
\left[(1-x) \prod_{j=1}^{p-1}(-1-j-j x)^{2 p}\right]_{x^{p^{2}}} \equiv\left[(1-x) \prod_{j=1}^{p-1}\left(-1-j-j x^{p}\right)^{2}\right]_{x^{p^{2}}} \equiv\left[\prod_{j=1}^{p-1}(-1-j-j y)^{2}\right]_{y^{p}} .
$$

On proceding as in the previous proof we find that

$$
v_{p^{2}+1} \equiv\left[\left(\frac{y^{p}-y}{1-y}\right)^{2}\right]_{y^{p}}=\left[\left(\frac{y}{1-y}\right)^{2}\right]_{y^{p}}=\left[\sum_{k \geq 1} k y^{k+1}\right]_{y^{p}} \equiv-1(\bmod p) .
$$

Finally, by Lemma 3. $v_{p^{2}+2}$ satisfies the same congruence as $v_{p^{2}+1}$ modulo $p$.

Lemma 10 Let $p$ be a prime. Then $v_{p^{2}+p+1} \equiv v_{p^{2}+p+2} \equiv-1(\bmod p)$.

Proof. We have

$$
\begin{aligned}
v_{p^{2}+p+1} & \equiv\left[(1-x)\left(x+x^{2}+\cdots+x^{p-1}\right)^{2 p+2}\right]_{x^{p^{2}+p}}(\bmod p) \\
& \equiv\left[(1-x)\left(x+x^{2}+\cdots+x^{p-1}\right)^{2}\left(x^{p}+\cdots+x^{p(p-1)}\right)^{2}\right]_{x^{p^{2}+p}}(\bmod p) \\
& \equiv\left[\left(x-x^{p}\right)\left(x+x^{2}+\cdots+x^{p-1}\right)\left(\sum_{k=1}^{\infty} x^{k p}\right)^{2}\right]_{x^{p^{2}+p}}(\bmod p) \\
& \equiv\left[\left(x^{2}+\cdots+x^{p}-x^{p+1}-\cdots-x^{2 p-1}\right) \sum_{k=0}^{\infty}(k+1) x^{k p}\right]_{x^{p^{2}-p}} \\
& \equiv\left[\sum_{k=0}^{\infty}(k+1) x^{(k+1) p}\right]_{x^{p^{2}-p}} \equiv-1(\bmod p) .
\end{aligned}
$$

Finally, by Lemma 3, $v_{p^{2}+p+2}$ satisfies the same congruence as $v_{p^{2}+p+1}$ modulo $p$.

The next lemma establishes part 6 of Theorem 1

Lemma 11 If $p$ is an odd prime, then $v_{\frac{p+3}{2}+i} \equiv 0(\bmod p)$ for $i=0, \ldots,(p-3) / 2$.

Proof. In case $i=0$, the result follows by Lemma 11 so assume that $i \geq 1$. On using that modulo $p$ the $j$ th term equals the $(j+p)$ th term, we find that, modulo $p$,

$$
v_{\frac{p+3}{2}+i} \equiv\left[4 i^{2}(1-x) \prod_{j=1}^{p-1}(2 i-j+j x) \prod_{j=1}^{2 i}(2 i-j+j x)\right]_{x^{(p+1) / 2+j}} .
$$

On invoking Lemma 5 and noting that $p>\frac{p+1}{2}+i$ we infer that

$$
v_{\frac{p+3}{2}+i} \equiv\left[4 i^{2}\left(x^{p}-x\right) \prod_{j=1}^{2 i}(2 i-j+j x)\right]_{x^{(p+1) / 2+i}} \equiv\left[-4 i^{2} \prod_{j=1}^{2 i}(2 i-j+j x)\right]_{x^{(p-1) / 2+j}} .
$$

Since $\operatorname{deg}\left(\prod_{j=1}^{2 i}(2 i-j+j x)\right)=2 i$ and $2 i<\frac{p-1}{2}+i$, the result follows.

The next lemma will be used in the proof of Lemma 13. 
Lemma 12 Define $A_{r}(x)$ and $B_{r}(x)$ recursively by

$$
\left\{\begin{array}{l}
A_{0}(x)=0, A_{r+1}(x)=\left(x+\ldots+x^{p-1}\right)^{r}-A_{r}(x) \\
B_{0}(x)=0, B_{r+1}(x)=-\left(x+\ldots+x^{p-1}\right)^{r}-B_{r}(x) .
\end{array}\right.
$$

Put $f_{r}(x)=(x-1)\left(1+x^{p}+\ldots+x^{p(p-1)}\right)\left(x+\ldots+x^{p-1}\right)^{r}$. Then

$$
f_{r}(x)=(-1)^{r}(x-1)\left(1+x^{p}+\ldots+x^{p(p-1)}\right)+x^{p^{2}} A_{r}(x)+B_{r}(x),
$$

where, for $r \geq 1$, the degree of $B_{r}(x)$ equals $(r-1)(p-1)$.

Proof. Easily follows on noting that

$$
\begin{aligned}
f_{r+1}(x) & =\left(1+\ldots+x^{p-1}\right) f_{r}(x)-f_{r}(x) \\
& =\left(x^{p^{2}}-1\right)\left(x+\ldots+x^{p-1}\right)^{r}-f_{r}(x) .
\end{aligned}
$$

The next lemma is part 7 of Theorem 1

Lemma 13 Let $p$ be an odd prime. Suppose that $0 \leq i \leq(p-3) / 2$ and $(p-1) / 2 \leq l \leq p$. Then $v_{l p+(p+3) / 2+i} \equiv 0(\bmod p)$.

Proof. Write $l=(p-1) / 2+k$ (thus $k$ assumes the values $0, \ldots,(p+1) / 2)$. Put $P_{2 i}(x)=(2 i)^{2 l+2} \prod_{j=1}^{2 i}(2 i-j+j x)$. Note that the degree of this polynomial equals $2 i$. Proceding as in Lemma 11] we infer that, modulo $p$,

$$
\begin{aligned}
v_{l p+\frac{p+3}{2}+i} & \equiv\left[(x-1)\left(x+\ldots+x^{p-1}\right)^{2 l+1} P_{2 i}(x)\right]_{x^{l p+(p+1) / 2+i}} \\
& \equiv\left[(x-1)\left(x^{p}+x^{2 p} \ldots+x^{p(p-1)}\right)\left(x+\ldots+x^{p-1}\right)^{2 k} P_{2 i}(x)\right]_{x^{l p+(p+1) / 2+i}} .
\end{aligned}
$$

We consider the case $l=p$ (that is $k=(p+1) / 2)$ first. Then

$$
\begin{aligned}
v_{l p+\frac{p+3}{2}+i} & \equiv\left[(x-1)\left(x^{p}+x^{2 p}+\ldots+x^{p(p-1)}\right)^{2}\left(x+\ldots+x^{p-1}\right) P_{2 i}(x)\right]_{x^{l p+(p+1) / 2+i}} \\
& =\left[\left(x^{p}-x\right)\left(x^{p}+x^{2 p}+\ldots+x^{p(p-1)}\right)^{2} P_{2 i}(x)\right]_{x^{l p+(p+1) / 2+i}}=0,
\end{aligned}
$$

where we used the observation that the polynomial in brackets has the form

$$
\sum_{k} P_{2 i}(x)\left(c_{k p} x^{k p}+c_{k p+1} x^{k p+1}\right),
$$

and that $1+2 i<(p+1) / 2+i \leq p-1$.

Thus we may assume that $l \leq p-1$. Notice that $l p+(p+1) / 2+i<p^{2}$. Furthermore, by Lemma 12 we have $\left[B_{2 k}(x) P_{2 i}(x)\right]_{x^{(l-1) p+(p+1) / 2+i}}=0$, as

$$
2 k-1+2 i<(l-1) p+\frac{p+1}{2}+i .
$$

Using this, (6) and Lemma 12 we infer that

$$
\begin{aligned}
v_{l p+\frac{p+3}{2}+i} & \equiv\left[(x-1)\left(x^{p}+\ldots+x^{p(p-1)}\right)\left(x+\ldots+x^{p-1}\right)^{2 k} P_{2 i}(x)\right]_{x^{l p+(p+1) / 2+i}} \\
& =\left[(x-1)\left(x^{p}+\ldots+x^{p^{2}}\right)\left(x+\ldots+x^{p-1}\right)^{2 k} P_{2 i}(x)\right]_{x^{l p+(p+1) / 2+i}}
\end{aligned}
$$




$$
\begin{aligned}
& =\left[(x-1)\left(1+\ldots+x^{p(p-1)}\right)\left(x+\ldots+x^{p-1}\right)^{2 k} P_{2 i}(x)\right]_{x^{(l-1) p+(p+1) / 2+i}} \\
& =\left[(x-1)\left(1+\ldots+x^{p(p-1)}\right) P_{2 i}(x)+B_{2 k}(x) P_{2 i}(x)\right]_{x^{(l-1) p+(p+1) / 2+i}} \\
& =\left[(x-1)\left(1+\ldots+x^{p(p-1)}\right) P_{2 i}(x)\right]_{x^{(l-1) p+(p+1) / 2+i}}=0 .
\end{aligned}
$$

This concludes the proof.

A further question concerning the distribution of $v_{n}$ modulo primes is how frequently certain residues appear. For example, is it true that the zero have density 1 ? Is it true that the non-zero entries are equidistributed? Questions like this can be answered for the middle binomial coefficient $\left(\begin{array}{c}2 k \\ k\end{array}\right)$, see e.g. [2, 16, 17]. The following lemma suggests that perhaps techniques from the latter papers can be used to investigate this issue.

Lemma 14 We have $v_{1+3 k} \equiv v_{2+3 k} \equiv \frac{1}{k+1}\left(\begin{array}{c}2 k \\ k\end{array}\right)(\bmod 3)$ and $v_{3 k} \equiv 0(\bmod 3)$.

Proof. By Lemma 1] we have $v_{3 k} \equiv 0(\bmod 3)$. By Lemma 3 we have $v_{1+3 k} \equiv$ $v_{2+3 k}(\bmod 3)$. We have, modulo 3 ,

$$
\begin{aligned}
v_{2+3 k} & \equiv\left[(1-x) \prod_{j=0}^{1+6 k}(1-j+j x)\right]_{x^{1+3 k}}=\left[(1-x)(-x(1+x))^{2 k} x\right]_{x^{1+3 k}} \\
& =\left[(1-x)(1+x)^{2 k}\right]_{x^{k}}=\left(\begin{array}{c}
2 k \\
k
\end{array}\right)-\left(\begin{array}{c}
2 k \\
k-1
\end{array}\right)=\frac{1}{k+1}\left(\begin{array}{c}
2 k \\
k
\end{array}\right) .
\end{aligned}
$$

This concludes the proof.

\subsection{The sequence $\left\{v_{n}\right\}_{n=1}^{\infty}$ modulo prime powers}

The proof of the next lemma was kindly communicated to us by Carl Pomerance.

Lemma 15 The polynomial $\prod_{i=0}^{p^{l}-1}(i x-i+j)\left(\bmod p^{l}\right)$, as a polynomial in $x$, depends only on the class $j(\bmod p)$ (ie. replacing $j$ by $j+k p$ would yield the same result).

Proof. Let $f_{j}(x)=\prod_{i=0}^{p^{r}-1}(i x+j)$. If $p \mid j$, then there are $p^{r-1}$ factors divisible by $p$ and $p^{r-1} \geq r$, so that $f_{j}(x) \equiv 0\left(\bmod p^{r}\right)$. So assume $p \nmid j$. Let $k$ be the inverse of $j$, so $j k \equiv 1\left(\bmod p^{r}\right)$. Then modulo $p^{r}$, we have $f_{j}(x) \equiv j^{p^{r}} f_{1}(x)$ (since the expression $i k$ runs over a complete residue system modulo $p^{r}$ as $i$ runs). Now say $j \equiv j_{1}(\bmod p)$, say $j_{1}=j+k p$. Using induction with respect to $r$ one then easily sees that $j_{1}^{p^{r}}=(j+k p)^{p^{r}} \equiv j^{p^{r}}\left(\bmod p^{r}\right)$, and we are done.

Proof of Theorem 2, Part 1. We have

$$
\begin{aligned}
v_{\frac{p+3}{2}}= & p^{2}\left[(1-x) \prod_{j=1}^{p-1}(p-j+j x)\right]_{x^{\frac{p-1}{2}}} \\
\equiv & p^{2}\left[(1-x) \prod_{j=1}^{p-1}(-j+j x)+(1-x) p \sum_{k=1}^{p-1} \prod_{\substack{j=1 \\
j \neq k}}^{p-1}(-j+j x)\right. \\
& \left.+(1-x) p^{2} \sum_{1 \leq k<r \leq p-1} \prod_{\substack{j=1 \\
j \neq k, r}}^{p-1}(-j+j x)\right]_{x} \frac{p-1}{2}\left(\bmod p^{5}\right)
\end{aligned}
$$




$$
\begin{aligned}
\equiv & -p^{2}(p-1) !\left[(x-1)^{p}+(x-1)^{p-1} p \sum_{k=1}^{p-1} \frac{1}{k}\right. \\
& \left.+(x-1)^{p-2} p^{2} \sum_{1 \leq k<r \leq p-1} \frac{1}{k r}\right]_{x^{\frac{p-1}{2}}}\left(\bmod p^{5}\right) \\
\equiv & -p^{2}\{(p-1) !\}\left[(x-1)^{p}\right]_{x^{\frac{p-1}{2}}}\left(\bmod p^{5}\right), \\
\equiv & -p^{2}\{(p-1) !\}\left(\begin{array}{c}
p \\
\frac{p-1}{2}
\end{array}\right)(-1)^{\frac{p+1}{2}}\left(\bmod p^{5}\right), \\
\equiv & 2 p^{3}\{(p-1) !\}(1-p)\left(\begin{array}{c}
p-1 \\
\frac{p-1}{2}
\end{array}\right)(-1)^{\frac{p-1}{2}}\left(\bmod p^{5}\right),
\end{aligned}
$$

where we used that $\sum_{k=1}^{p-1} 1 / k \equiv 0\left(\bmod p^{2}\right)$ (this is Wolstenholme's theorem 12, Theorem 115]) and $\sum_{1 \leq k<r \leq p-1} 1 / k r \equiv 0(\bmod p)$. To see the latter congruence note that

$$
(p-1) ! \sum_{1 \leq k<r \leq p-1} \frac{1}{k r}=\left[\prod_{j=1}^{p-1}(x-j)\right]_{x^{p-3}} \equiv\left[x^{p-1}-1\right]_{x^{p-3}}=0(\bmod p) .
$$

Now it is an easy consequence of Eisenstein's congruence (1859), see [12, Theorem 132], which states that

$$
\frac{2^{p-1}-1}{p} \equiv 1+\frac{1}{3}+\frac{1}{5}+\cdots+\frac{1}{p-2}(\bmod p)
$$

that (see ibid. Theorem 133) $\left(\begin{array}{c}p \\ (p-1) / 2\end{array}\right)(-1)^{\frac{p-1}{2}} \equiv 4^{p-1}\left(\bmod p^{2}\right)$. (Indeed, by Morley's congruence (1895), cf. [4, this congruence is even valid modulo $p^{3}$.) We thus finally infer that $v_{\frac{p+3}{2}} \equiv 2 p^{3}(1-p)\{(p-1) !\} 4^{p-1}\left(\bmod p^{5}\right)$, which of course implies that $v_{\frac{p+3}{2}} \equiv$ $-2 p^{3}\left(\bmod p^{4}\right)$.

Part 2. We have the formal series identity

$$
\frac{1}{(1-x)^{2 r}}=\sum_{k=0}^{\infty}\left(\begin{array}{c}
k+2 r-1 \\
2 r-1
\end{array}\right) x^{k}
$$

Note that

$$
\left[(x-1)^{-2 r}\right]_{x^{\frac{p-1}{2}-s+r p}} \equiv \frac{(1-2 s)_{2 r-1}}{2^{2 r-1}(2 r-1) !}(\bmod p) .
$$

Using the latter congruence we find that, modulo $p^{2 r+3}$.

$$
\begin{aligned}
v_{\frac{p+3}{2}+r p} & =\left[(1-x) \prod_{j=0}^{(2 r+1) p}((2 r+1) p-j+j x)\right]_{x^{\frac{p+1}{2}+r p}} \\
& \equiv p^{2 r+2}\left[(1-x) \prod_{j=0}^{2 r+1}(2 r+1-j+j x) \prod_{\substack{j=0 \\
p \nmid j}}^{(2 r+1) p}(-j+j x)\right]_{x^{\frac{p+1}{2}+r p}} \\
& \equiv(2 r+1)^{2} p^{2 r+2}\left[\left(\sum_{j=0}^{2 r} b_{j, r} x^{j}\right)(x-1)^{(2 r+1) p-2 r}\right]_{x^{(p-1) / 2+r p}} \\
& \equiv(2 r+1)^{2} p^{2 r+2}\left[\left(\sum_{j=0}^{2 r} b_{j, r} x^{j}\right)(x-1)^{(2 r+1) p} \sum_{k=0}^{\infty}\left(\begin{array}{c}
k+2 r-1 \\
2 r-1
\end{array}\right) x^{k}\right]_{x^{(p-1) / 2+r p}}
\end{aligned}
$$




$$
\begin{aligned}
& \equiv-(2 r+1)^{2} p^{2 r+2}\left[\left(\sum_{j=0}^{2 r} b_{j, r} x^{j}\right) \sum_{l=0}^{r} x^{l p}(-1)^{l} \sum_{k=0}^{\infty}\left(\begin{array}{c}
k+2 r-1 \\
2 r-1
\end{array}\right) x^{k}\right]_{x^{(p-1) / 2+r p}} \\
& \equiv-(2 r+1)^{2} p^{2 r+2} \sum_{l=0}^{r}\left(\begin{array}{c}
2 r+1 \\
l
\end{array}\right)(-1)^{l} \sum_{j=0}^{2 r} b_{j, r}\left(\begin{array}{c}
\frac{p-1}{2}-j+(r-l) p+2 r-1 \\
2 r-1
\end{array}\right) \\
& \equiv-\frac{(2 r+1)^{2} p^{2 r+2}}{2^{2 r-1}(2 r-1) !} \sum_{l=0}^{r}\left(\begin{array}{c}
2 r+1 \\
l
\end{array}\right)(-1)^{l} \sum_{j=0}^{2 r} b_{j, r}((1-2 j))_{2 r-1} \\
& \equiv \frac{(-1)^{r-1}(2 r+1)^{2}}{2^{2 r-1}(2 r-1) !}\left(\begin{array}{c}
2 r \\
r
\end{array}\right) p^{2 r+2} \sum_{j=0}^{2 r} b_{j, r}((1-2 j))_{2 r-1} \\
& =C_{r} p^{2 r+2},
\end{aligned}
$$

where in the one but last step we used the identity

$$
(-1)^{r}\left(\begin{array}{c}
2 r \\
r
\end{array}\right)=\sum_{l=0}^{r}\left(\begin{array}{c}
2 r+1 \\
l
\end{array}\right)(-1)^{l},
$$

which is obtained by comparing the coefficient of $x^{r}$ of both sides of the identity $(1-x)^{-1}(1-x)^{2 r+1}=(1-x)^{2 r}$. This finishes the proof.

\subsection{The sequence $\left\{v_{n}\right\}_{n=1}^{\infty}$ modulo powers of two}

Before we can consider the sequence modulo powers of two we need some preparatory lemmas.

Lemma 16 If $j$ is odd, then $\prod_{i=0}^{2^{q}-1}(i x-i+j)^{2} \equiv x^{2^{q}}\left(\bmod 2^{q}\right)$.

Proof. By induction with respect to $q$. For $q=1$ the result is obvious. Assume the result is established for $1 \leq q \leq q_{1}$. We write

$$
\prod_{i=0}^{2^{q_{1}+1}-1}(i x-i+j)^{2}=\prod_{i=0}^{2^{q_{1}}-1}(i x-i+j)^{2} \prod_{i=2^{q_{1}}}^{2^{q_{1}+1}-1}(i x-i+j)^{2}=P_{1}(x) P_{2}(x),
$$

say. Note that $P_{1}(x) \equiv P_{2}(x)\left(\bmod 2^{q_{1}}\right)$. The induction hypothesis thus implies that we can write $P_{1}(x)=x^{2^{q_{1}}}+2^{q_{1}} f_{1}(x)$ and $P_{2}(x)=x^{2^{q_{1}}}+2^{q_{1}} f_{2}(x)$. Since $(i x-i+j)^{2} \equiv$ $\left(\left(i+2^{q_{1}}\right) x-\left(i+2^{q_{1}}\right)+j\right)^{2}\left(\bmod 2^{q_{1}+1}\right)$, it even follows that $P_{1}(x) \equiv P_{2}(x)\left(\bmod 2^{q_{1}+1}\right)$, from which we infer that $f_{1}(x) \equiv f_{2}(x)(\bmod 2)$ and hence $f_{1}(x)+f_{2}(x) \equiv 0(\bmod 2)$. It follows that modulo $2^{q_{1}+1}$ the product under consideration equals

$$
P_{1}(x) P_{2}(x)=\left(x^{2^{q_{1}}}+2^{q_{1}} f_{1}(x)\right)\left(x^{2^{q_{1}}}+2^{q_{1}} f_{2}(x)\right)=x^{2^{q_{1}+1}}\left(\bmod 2^{q_{1}+1}\right) .
$$

This concludes the proof.

In the course of the above proof we have showed that

$$
\prod_{i=0}^{2^{q}-1}(i x-i+j)^{2} \equiv \prod_{i=2^{q}}^{2^{q+1}-1}(i x-i+j)^{2}\left(\bmod 2^{q+1}\right) .
$$

The next result shows that the same identity holds true for the 'square roots'. Using this the 'square root' of the left hand side of Lemma 16 can be computed (Lemma 18). 
Lemma 17 Let $j$ be odd and $q \geq 2$. Then

$$
\prod_{i=0}^{2^{q}-1}(i x-i+j) \equiv \prod_{i=2^{q}}^{2^{q+1}-1}(i x-i+j)\left(\bmod 2^{q+1}\right) .
$$

Proof. It is an easy observation that, modulo 2 , we have for $0 \leq k \leq 2^{q}-1$ that

$$
\prod_{a=0, a \neq k}^{2^{q}-1}(j-a+a x) \equiv \begin{cases}x^{2^{q-1}-1} & \text { if } k \text { is odd } \\ x^{2^{q-1}} & \text { if } k \text { is even }\end{cases}
$$

Using this identity we find that, modulo $2^{q+1}$,

$$
\begin{aligned}
\prod_{i=2^{q}}^{2^{q+1}-1}(i x-i+j) & =\prod_{i=0}^{2^{q}-1}\left(i x-i+j+2^{q}(x-1)\right) \\
& \equiv \prod_{i=0}^{2^{q}-1}(i x-i+j)+2^{q}(x-1) \sum_{k=0}^{2^{q}-1} \prod_{i=0, i \neq k}^{2^{q}-1}(i x-i+j) \\
& \equiv \prod_{i=0}^{2^{q}-1}(i x-i+j)+2^{q}(x-1)\left(x^{2^{q-1}} \sum_{2 \mid k}^{2^{q}-2} 1+x^{2^{q-1}-1} \sum_{2 \nmid k}^{2^{q}-1} 1\right) \\
& \equiv \prod_{i=0}^{2^{q}-1}(i x-i+j)+2^{q}(x-1)\left(x^{2^{q-1}} 2^{q-1}+x^{2^{q-1}-1} 2^{q-1}\right) \\
& \equiv \prod_{i=0}^{2^{q}-1}(i x-i+j) .
\end{aligned}
$$

This finishes the proof.

Lemma 18 Let $j$ be odd and $q \geq 3$. We have

$$
\prod_{i=0}^{2^{q}-1}(i x-i+j) \equiv x^{2^{q-1}-2}\left[2^{q-1}\left(x^{4}+x^{3}+x+1\right)+x^{2}\right]\left(\bmod 2^{q}\right) .
$$

Proof. Similar to that of Lemma [16, but with the difference that instead of the equality $P_{1}(x) \equiv P_{2}(x)\left(\bmod 2^{q+1}\right)$, we use Lemma 17

Remark. By Lemma 15] it suffices to work in the proofs of Lemma 16, 17 and 18 with $j=1$.

The next result established part of parts 8 and 9 of Theorem 1]

Lemma 19 For $q \geq 1$ we have $v_{2^{q}} \equiv-1\left(\bmod 2^{q}\right)$.

Proof. Put $P_{q}(x)=(1-x) \prod_{j=0}^{2^{q+1}-3}(-3-j+j x)$. We want to compute the coefficient of $x^{2^{q}-1}$ in $P_{q}(x)$ modulo $2^{q}$. On invoking Lemma [16] one finds that

$$
P_{q}(x)(1+2 x)(2+x) \equiv x^{2^{q}}(1-x)\left(\bmod 2^{q}\right),
$$


from which we infer that

$$
\begin{aligned}
P_{q}(x) & \equiv x^{2^{q}-q}(1-x) \sum_{k=0}^{\infty}(-2)^{k} x^{k} \sum_{r=0}^{q-1}(-2)^{q-1-r} x^{r}\left(\bmod 2^{q}\right) \\
& \equiv x^{2^{q}-q}(1-x) \sum_{m=0}^{2 q-2} a_{m} x^{m} \equiv x^{2^{q}-q} \sum_{m=0}^{2 q-1} b_{m} x^{m}\left(\bmod 2^{q}\right),
\end{aligned}
$$

where

$a_{m} \equiv\left\{\begin{array}{ll}-(-2)^{q-1-m} / 3 & \text { if } 0 \leq m \leq q-1 ; \\ -(-2)^{-q+1+m} / 3 & \text { if } q \leq m \leq 2 q-2\end{array}\right.$ and $b_{m} \equiv \begin{cases}-(-2)^{q-1-m} & \text { if } 0 \leq m \leq q-1 \\ (-2)^{m-q} & \text { if } q \leq m \leq 2 q-1\end{cases}$

Thus $v_{2^{q}} \equiv b_{q-1} \equiv-1\left(\bmod 2^{q}\right)$.

Recall that we defined $v_{0}=-1$. The reason for this is that this definition allows us to also formulate the next lemma, which together with Lemma 23] is part 8 of Theorem 11. with $j=0$.

Lemma 20 (Periodicity.) Suppose that $i, k \geq 0$. We have $v_{k 2^{q}+i} \equiv v_{i}\left(\bmod 2^{q}\right)$.

Proof. First assume that $i \geq 2$. Note that

$$
v_{k 2^{q}+i} \equiv\left[(1-x) \prod_{j=0}^{2^{q}-1}(2 i-3-j+j x)^{2 k} \prod_{j=0}^{2 i-3}(2 i-3-j+j x)\right]_{x^{k 2^{q}+i-1}}\left(\bmod 2^{q}\right) .
$$

By lemma 16, the first product equals $x^{k 2^{q}} \bmod 2^{q}$. Thus

$$
v_{k 2^{q}+i} \equiv\left[(1-x) \prod_{j=0}^{2 i-3}(2 i-3-j+j x)\right]_{x^{i-1}} \equiv v_{i}\left(\bmod 2^{q}\right) .
$$

In order to deal with the case $i=1$, we note that, using Lemma 3, $v_{k 2^{q}+1} \equiv v_{k 2^{q}+2} \equiv$ $v_{2} \equiv v_{1}\left(\bmod 2^{q}\right)$. In case $i=0$ one finds proceding as above that, for $k \geq 1, v_{k 2^{q}} \equiv$ $v_{2^{q}}\left(\bmod 2^{q}\right)$. On invoking Lemma 19$]$ it then follows that $v_{k 2^{q}} \equiv v_{2^{q}} \equiv v_{0}\left(\bmod 2^{q}\right)$.

The next result yields a part of part 9 of Theorem 1

Lemma 21 Suppose that $q \geq 1$. Then $v_{2^{q-1}} \equiv 2^{q-1}-1\left(\bmod 2^{q}\right)$.

Proof. Similar to that of Lemma 19, For $q \leq 3$ one verifies the claim numerically. So assume $q \geq 4$. We want to compute the coefficient of $x^{2^{q-1}-1}$ in $P_{q-1}(x)$ modulo $2^{q}$. On invoking Lemma 18 one finds that

$$
P_{q-1}(x)(1+2 x)(2+x) \equiv x^{2^{q-1}-2}\left[2^{q-1}\left(x^{4}+x^{3}+x+1\right)+x^{2}\right]\left(\bmod 2^{q}\right),
$$

whence $P_{q-1}(x) \equiv x^{2^{q-1}-q-2}\left(\sum_{m=0}^{2 q-1} b_{m} x^{m}\right)\left[2^{q-1}\left(x^{4}+x^{3}+x+1\right)+x^{2}\right]\left(\bmod 2^{q}\right)$. (Note that the assumption $q \geq 4$ implies that $2^{q-1}-q-2 \geq 0$.) Thus modulo $2^{q}$ the coefficient of $x^{2^{q-1}-1}$, that is $v_{2^{q-1}}$, equals

$$
v_{2^{q-1}} \equiv b_{q-1}+2^{q-1}\left(b_{q-3}+b_{q-2}+b_{q}+b_{q+1}\right)
$$




$$
\equiv-1+2^{q-1}(-4+2-2+1) \equiv 2^{q-1}-1
$$

This completes the proof.

The next result with $i=0,1$ and 2 yields a part of part 9 of Theorem 11. It also yields part 10 of Theorem 1

Lemma 22 For $i \geq 0$ and $q \geq 2$ we have $v_{2^{q-1}+i} \equiv v_{i}+2^{q-1}\left(\bmod 2^{q}\right)$.

Proof. For $q=2$ one checks the result numerically and so we may assume $q \geq 3$. For $i=0$ the result follows by Lemma 21]. Note that, a priori, $v_{2^{q-1}+i} \equiv v_{i}\left(\bmod 2^{q-1}\right)$ and so either $v_{2^{q-1}+i} \equiv v_{i}\left(\bmod 2^{q}\right)$ or $v_{2^{q-1}+i} \equiv v_{i}+2^{q-1}\left(\bmod 2^{q}\right)$. Let us first assume that $i \geq 2$. The idea of the proof is to use Lemma 17 to write $v_{2^{q-1}+i} \equiv v_{i}+2^{q-1}\left[f_{q, i}(x)\right]_{x^{2^{q-1}+i-1}}\left(\bmod 2^{q}\right)$. An easy computation then shows that $\left[f_{q, i}(x)\right]_{x^{2^{q-1}+i-1}}$ is odd, thus finishing the proof.

More precisely, one first notes that, modulo $2^{q}$,

$$
\begin{aligned}
v_{2^{q-1}+i} & \equiv\left[(1-x) \prod_{j=0}^{2^{q}-1}(2 i-3-j+j x) \prod_{j=2^{q}}^{2^{q}+2 i-3}(2 i-3-j+j x)\right]_{x^{2^{q-1}+i-1}} \\
& \equiv\left[(1-x) \prod_{j=0}^{2 i-3}(2 i-3-j+j x) \prod_{j=0}^{2^{q}-1}(2 i-3-j+j x)\right]_{x^{2^{q-1}+i-1}} \\
& \equiv\left[(1-x) x^{2^{q-1}-2}\left(2^{q-1}\left(x^{4}+x^{3}+x+1\right)+x^{2}\right) \prod_{j=0}^{2 i-3}(2 i-3-j+j x)\right]_{x^{2^{q-1}+i-1}} \\
& \equiv v_{i}+2^{q-1}\left[(1-x)\left(x^{4}+x^{3}+x+1\right) \prod_{j=0}^{2 i-3}(2 i-3-j+j x)\right]_{x^{i+1}} \\
& \equiv v_{i}+2^{q-1}\left[(1-x)\left(x^{4}+x^{3}+x+1\right) x^{i-1}\right]_{x^{i+1}}
\end{aligned}
$$

Since $\left[(1-x)\left(x^{4}+x^{3}+x+1\right) x^{i-1}\right]_{x^{i+1}}=\left[(1-x)\left(x^{4}+x^{3}+x+1\right)\right]_{x^{2}}=-1$ is odd, the result follows for $j \geq 2$. On combining this result for $i=2$ and Lemma 3 we find that $v_{2^{q-1}+1} \equiv v_{2^{q-1}+2} \equiv v_{2}=v_{1}\left(\bmod 2^{q}\right)$. Thus the result follows for every $j \geq 0$.

Using induction and the latter lemma one then easily infers the following result which, together with Lemma 20, gives part 8 of Theorem 1.

Lemma 23 (Equidistribution.) Let $q \geq 1$. For every odd integer a there are precisely two integers $1 \leq j_{1}<j_{2} \leq 2^{q}$ such that $v_{j_{1}} \equiv a\left(\bmod 2^{q}\right)$ and $v_{j_{2}} \equiv a\left(\bmod 2^{q}\right)$.

\subsection{On a result of Paolo Dominici}

Let $S_{k}\left(x_{1}, \ldots, x_{r}\right)$ denote the $k$ th elementary symmetric function in $r$ variables, i.e. $S_{1}\left(x_{1}, \ldots, x_{r}\right)=x_{1}+\ldots+x_{r}, S_{2}\left(x_{1}, \ldots, x_{r}\right)=x_{1} x_{2}+x_{1} x_{3}+\ldots+x_{r-1} x_{r}$, etc.. Paolo Dominici [7] states the following result for $v_{n}$ without reference.

Theorem 3 For $1 \leq i \leq 2 n-4$ we put $y_{i}=i /(2 n-3-i)$. Then

$$
v_{n}=(2 n-3)^{2}(2 n-4) !\left\{S_{n-2}\left(y_{1}, \ldots, y_{2 n-4}\right)-S_{n-1}\left(y_{1}, \ldots, y_{2 n-4}\right)\right\} \text {. }
$$

We will now derive this result from (3). We need two lemmas 
Lemma 24 Let $L_{1}(x), \ldots, L_{r}(x)$ be linear polynomials, then

$$
\frac{1}{m !} \frac{d^{m}}{d x^{m}}\left\{L_{1}(x) \cdots L_{r}(x)\right\}=S_{m}\left(\frac{L_{1}^{\prime}(x)}{L_{1}(x)}, \ldots, \frac{L_{r}^{\prime}(x)}{L_{r}(x)}\right) L_{1}(x) \cdots L_{r}(x) .
$$

Another observation we need is the following:

Lemma 25 Let $x_{1}, \ldots, x_{r}$ be distinct non-zero elements such that $x_{1} \cdot x_{2} \cdots x_{r}=1$ and $\left\{x_{1}, \ldots, x_{r}\right\}=\left\{\frac{1}{x_{1}}, \ldots, \frac{1}{x_{r}}\right\}$. Then $S_{r-k}\left(x_{1}, \ldots, x_{r}\right)=S_{k}\left(x_{1}, \ldots, x_{r}\right)$, with $1 \leq r \leq k$.

Proof. Note that $S_{r-k}\left(x_{1}, \ldots, x_{r}\right)=S_{k}\left(\frac{1}{x_{1}}, \ldots, \frac{1}{x_{r}}\right) x_{1} \cdots x_{r}=S_{k}\left(x_{1}, \ldots, x_{r}\right)$, where in the derivation of the first equality we used the assumption that $x_{i} \neq 0$ and in that of the second the remaining assumptions.

Corollary 1 For $1 \leq k \leq 2 n-5$ we have $S_{k}\left(y_{1}, \ldots, y_{2 n-4}\right)=S_{2 n-4-k}\left(y_{1}, \ldots, y_{2 n-4}\right)$.

Proof of Theorem 3. Put $P_{n}(x)=\prod_{j=1}^{2 n-4}(2 n-3-j+j x)$. By definition we have

$$
v_{n}=\left[(1-x) \prod_{j=0}^{2 n-3}(2 n-3-j+j x)\right]_{x^{n-1}}=(2 n-3)^{2}\left[(1-x) P_{n}(x)\right]_{x^{n-2}} .
$$

Thus,

$$
v_{n}=(2 n-3)^{2}\left\{\left[P_{n}(x)\right]_{x^{n-2}}-\left[P_{n}(x)\right]_{x^{n-3}}\right\} .
$$

On noting that $\left[P_{n}(x)\right]_{x^{m}}=\left.\frac{1}{m !} \frac{d^{m}}{d x^{m}} P_{n}(x)\right|_{x=0}$, we obtain on invoking Lemma 24 that

$$
\left[P_{n}(x)\right]_{x^{m}}=(2 n-4) ! S_{m}\left(y_{1}, \ldots, y_{i}, \ldots, y_{2 n-4}\right) .
$$

Combining (18) with (17) yields that

$$
v_{n}=(2 n-3)^{2}(2 n-4) !\left\{S_{n-2}\left(y_{1}, \ldots, y_{2 n-4}\right)-S_{n-3}\left(y_{1}, \ldots, y_{2 n-4}\right)\right\},
$$

or, on invoking Corollary 1.

$$
v_{n}=(2 n-3)^{2}(2 n-4) !\left\{S_{n-2}\left(y_{1}, \ldots, y_{2 n-4}\right)-S_{n-1}\left(y_{1}, \ldots, y_{2 n-4}\right)\right\},
$$

This concludes the proof.

From the above proof we infer that we may alternatively define $v_{n}$ by

$$
v_{n}=\left[(x-1) \prod_{j=0}^{2 n-3}(2 n-3-j+j x)\right]_{x^{n}} .
$$

We leave it to the reader to use the observation that $P(x):=\prod_{j=1}^{2 n-4}(2 n-3-j+j x)$ is selfreciprocal, i.e. satisfies $P(1 / x) x^{2 m-4}=P(x)$ to infer (9) directly from (3).

Many of the congruences can be also proved using Theorem 3. As an example we will show that if $p$ is an odd prime, then $v_{3(p+1) / 2} \equiv-81 p^{4}\left(\bmod p^{5}\right)$. This is the case $r=1$ of part 2 of Theorem 2 . 
Proof of part 2 of Theorem 2 in case $r=1$. Set $n=3(p+1) / 2$. Note that $(2 n-3)^{2}(2 n-$ $4) ! \equiv-18 p^{4}\left(\bmod p^{5}\right)$. It thus remains to be proven that the expression in braces in Theorem 3 equals $9 / 2$ modulo $p$. It turns out to be a little easier to work with $w_{i}=-y_{i}$. Note that $(-1)^{r} S_{r}\left(w_{1}, \ldots, w_{3 p-1}\right)=S_{r}\left(y_{1}, \ldots, y_{3 p-1}\right)$. We have $w_{i}=i /(i-3 p)$ for $1 \leq i \leq 3 p-1$. Thus $w_{p}=-1 / 2, w_{2 p}=-2$ and the remaining $w_{i}$ satisfy $w_{i} \equiv 1(\bmod p)$. Hence $S_{r}\left(w_{1}, \ldots, w_{3 p-1}\right) \equiv S_{r}(-1 / 2,-2,1,1, \ldots, 1)(\bmod p)$, where $2 \leq r \leq 3 p-1$. In the symmetric function $S_{r}\left(z_{1}, \ldots, z_{3 p-1}\right)$ there are $\left(\begin{array}{c}3 p-3 \\ r\end{array}\right)$ terms containing neither $z_{1}$ nor $z_{2}$. There are $\left(\begin{array}{c}3 p-3 \\ r-1\end{array}\right)$ terms containing $z_{1}$, but not $z_{2}$. Finally there are $\left(\begin{array}{c}3 p-3 \\ r-2\end{array}\right)$ terms containing both $z_{1}$ and $z_{2}$. It follows that, modulo $p$,

$$
(-1)^{r} S_{r}\left(y_{1}, \ldots, y_{3 p-1}\right) \equiv S_{r}\left(-\frac{1}{2},-2,1, \ldots, 1\right)=\left(\begin{array}{c}
3 p-3 \\
r
\end{array}\right)-\left(2+\frac{1}{2}\right)\left(\begin{array}{c}
3 p-3 \\
r-1
\end{array}\right)+\left(\begin{array}{c}
3 p-3 \\
r-2
\end{array}\right) .
$$

Modulo $p$ we have

$$
\begin{aligned}
(-1)^{n} & \left\{S_{n-2}\left(y_{1}, \ldots, y_{2 n-4}\right)-S_{n-1}\left(y_{1}, \ldots, y_{2 n-4}\right)\right\} \\
\equiv & \left(\begin{array}{c}
3 p-2 \\
n-1
\end{array}\right)-\frac{5}{2}\left(\begin{array}{c}
3 p-2 \\
n-2
\end{array}\right)+\left(\begin{array}{c}
3 p-2 \\
n-3
\end{array}\right) \\
\equiv & 2\left(\begin{array}{c}
p-2 \\
n-p-1
\end{array}\right)-5\left(\begin{array}{c}
p-2 \\
n-p-2
\end{array}\right)+2\left(\begin{array}{c}
p-2 \\
n-p-3
\end{array}\right) \\
\equiv & {\left[2\left(\begin{array}{c}
p-2 \\
n-p-1
\end{array}\right)+4\left(\begin{array}{c}
p-2 \\
n-p-2
\end{array}\right)+2\left(\begin{array}{c}
p-2 \\
n-p-3
\end{array}\right)\right]-9\left(\begin{array}{c}
p-2 \\
n-p-2
\end{array}\right) } \\
\equiv & {\left[2\left(\begin{array}{c}
p \\
n-p-1
\end{array}\right)\right]-9\left(\begin{array}{c}
p-2 \\
n-p-2
\end{array}\right) } \\
\equiv & -9\left(\begin{array}{c}
p-2 \\
n-p-2
\end{array}\right) \equiv(-1)^{n} 9(n-p-1) \equiv(-1)^{n} \frac{9}{2} .
\end{aligned}
$$

This completes the proof.

\section{Asymptotics}

Given a sequence of coefficients, there are many things we would like to know about it. Apart from the search for a generating function and for a recursion formula, an interesting question is the asymptotic behaviour. We remind the reader that candidate Fourier series for modular forms of weight $2 k$ for $S L(2, \mathbb{Z})$ must have coefficients growing like $n^{2 k-1}$ (and $n^{k}$ for cusp forms).

In our case, without prior knowledge of the alternative definition (3), we only managed to compute the first 80 values of $v_{n}$ using the Schubert package for intersection theory [13] or the first 225 values using the rational function (with the dummy variables $\left.w_{i}\right)$. Numerically, it is readily seen that the leading term for the $v_{n}$ is $e^{2 n \log n}$. As this is strongly reminiscent of the behaviour of $(2 n) !=\exp \left(2 n \log (2 n)-2 n+\frac{1}{2} \log 2 n+\right.$ $\left.\frac{1}{2} \log 2 \pi+O\left(\frac{1}{n}\right)\right)$, we rather study the behaviour of $\log \frac{v_{n}}{(2 n) !}$ and find now the leading term to be $2 n$. Subtracting it, we find the next-to-leading term to be $-4 \log n$, easily verified by applying $n \partial_{n}$ (ie. taking subsequent differences and multiplying by $n$ ). The next term is a constant, $C=-5.62 \ldots$, which we find difficult to recognize. We have learnt from Don Zagier a smart technique which enables to determine a large number of digits of $C$; we present it below under the name of $\operatorname{asymp}_{k}$ trick. 


\subsection{The $\operatorname{asymp}_{k}$ trick}

Assume we are given numerically a few hundred terms of a sequence $s=\left\{s_{n}\right\}_{n \in \mathbb{N}}$ which we believe has an asymptotic expansion goes in inverse powers of $n$, ie.

$$
s_{n} \sim c_{0}+\frac{c_{1}}{n}+\frac{c_{2}}{n^{2}}+\ldots
$$

Goal: determine the coefficients $c_{i}$ numerically.

Trick: Choose some moderate value of $k$ (say $k=8$ ) and define a new sequence $s^{(k)}$ as $\frac{1}{k !} \Delta^{k} N^{k} s$, where $\Delta$ is the difference operator $(\Delta u)_{n}=u_{n}-u_{n-1}$ and $N$ the multiplication operator $(N u)_{n}=n u_{n}$, i.e.,

$$
s_{n}^{(k)}=\sum_{j=0}^{k} \frac{(-1)^{j}}{j !(k-j) !}(n-j)^{k} s_{n-j} .
$$

For $n$ large we have (assuming the above asymptotic expansion for $s$ itself)

$$
s_{n}^{(k)}=c_{0}+(-1)^{k} \frac{c_{k+1}}{n^{k+1}}+(-1)^{k} \frac{\left((k+1) c_{k+2}-\left(\begin{array}{c}
k+1 \\
2
\end{array}\right) c_{k+1}\right)}{n^{k+2}}+\ldots
$$

Thus, while $s_{n}$ approximates $c_{0}$ only to within an accuracy $O\left(n^{-1}\right), s_{n}^{(k)}$ approximates it to the much better accuracy $O\left(n^{-k}\right)$, so we obtain a very good approximation for $c_{0}$. Call this operation $\operatorname{asymp}_{k}$. The further coefficients $c_{i}$ are then obtained inductively: if $c_{0}, \ldots, c_{i-1}$ are known to high precision, we get $c_{i}$ by applying $\operatorname{asymp}_{k}$ to the sequence $n^{i}\left(s_{n}-c_{0}-\cdots-c_{i-1} / n^{i-1}\right)=c_{i}+c_{i+1} / n+\cdots$.

The crucial point in the success of $\operatorname{asymp}_{k}$ is that the operator $\Delta^{k}$ sends $n^{k}$ to $k$ ! and kills polynomials of degree $<k$, so that all the intermediate terms of the expansion of $s_{n}$ between $c_{0}$ and $c_{k} n^{-k}$ disappear.

Variants of $\operatorname{asymp}_{k}$ allow one to deal for example with asymptotic expansions of the form

(I) $s_{n} \sim A \log n+c_{0}+c_{1} / n+c_{2} / n^{2}+\cdots$

(II) $s_{n} \sim B n+A \log n+c_{0}+c_{1} / n+c_{2} / n^{2}+\cdots$

(III) $s_{n} \sim A n^{\lambda}\left(1+c_{1} / n+c_{2} / n^{2}+\cdots\right)$

In case (I) we can apply $\operatorname{asymp}_{k}$ to the sequence $n\left(s_{n+1}-s_{n}\right)$, which has the form $A+c_{1}^{\prime} / n+c_{2}^{\prime} / n^{2}+\cdots$, to obtain $A$ to high precision, after which we apply the original

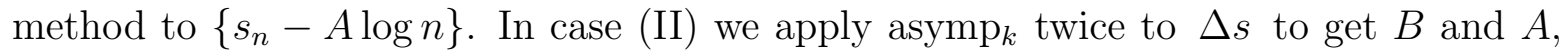
and then subtract (our approximation for) $B \log n+A$ from $s_{n}$ and apply the standard version. For case (III) we can either look at $\left\{\log s_{n}\right\}$ and apply variant (I) or else apply $\operatorname{asymp}_{k}$ to $\left\{n\left(s_{n+1} / s_{n}-1\right)\right\}$ to get $\lambda$ and then apply the standard method to $\left\{s_{n} / n^{\lambda}\right\}$.

Remark 1. Applying the operation $\operatorname{asymp}_{k}$ with suitably chosen $k$ gives a rapidly convergent sequence $s^{(k)}$. To estimate how many decimals are probably correct, we look at some relatively widely spaced elements of this sequence (e.g., the terms $s_{n}^{(k)}$ with $n=300,400,500$ if we know 500 terms of the sequence $s$ ) and see how many of their digits agree. 
Remark 2. One also has to experiment to find the optimal choice of $k$. Typically one uses $k=5$ if one knows 200 terms of $s$ and $k=8$ if one knows 1000 terms. This suggests that perhaps $k \approx \log N$ is a good choice for a generic sequence with $N$ computed terms.

Remark 3. The $\operatorname{asymp}_{k}$ trick was first described in a paper of Zagier [21]. Here he considers the Stoimenov numbers $\xi_{D}$ which bound the number $V(D)$ of linearly indepedent Vassiliev invariants of degree $D$. Stoimenov himself thought that $\xi_{D}$ behaves 'something like $D ! / 1.5^{D}$ '. Calculating the values up to $D=200$ and applying a variation of $\operatorname{asymp}_{k}$ suggested an asymptotic formula of the form

$$
\xi_{D} \sim \frac{D ! \sqrt{D}}{\left(\pi^{2} / 6\right)^{D}}\left(C_{0}+\frac{C_{1}}{D}+\frac{C_{2}}{D^{2}}+\cdots\right),
$$

with $C_{0} \approx 2.704332490062429595, C_{1} \approx-1.52707$ and $C_{2} \approx-0.269009$. Subsequently Zagier was able to prove this with explicitly computable constants $C_{i}$. In particular, $C_{0}=12 \sqrt{3} \pi^{-5 / 2} e^{\pi^{2} / 12}$, which agrees to the accuracy given above with the empirically obtained value.

\subsection{Application to the asymptotics of $v_{n}$}

In our case of sequence $v_{n}$ of lines in a hypersurface of $\mathbb{P}^{n}$, the coefficients $c_{0}=$ : $C$ is difficult to recognize, but all other coefficients, $c_{1}, c_{2}, \ldots$ are rational numbers which we easily recognize from a sufficient number of digits. Once the first few rational coefficients have been found and the corresponding terms subtracted from the sequence $s$, the constant term $C$ can be obtained with 30 digits, say. This is enough to feed to the PARI software and apply the function $\operatorname{lindep}([\mathrm{C}, 1, \log (\mathrm{Pi}), \log (2), \log (3)])$ to find a rational linear combination of $C$ in terms of a given basis (educated guess). The result, equivalent to (4) is:

$$
\log \frac{v_{n}}{(2 n) !}=2 n-4 \log n+C+\frac{11}{6 n}+\frac{141}{160 n^{2}}+\ldots,
$$

where $C:=-3-\log \pi-\frac{3}{2} \log \frac{8}{3}$. In the appendix we present a proof by Don Zagier of this asymptotic formula.

\section{Comparison with two other sequences}

As a matter of curiosity, we now compare our results so far with similar results from two other sequences of enumerative geometry. We shall see that the first case has quite similar features, while the second case is more intricate.

\subsection{Numbers of plane rational curves}

One sequence of integers from enumerative geometry is $n_{d}$, the number of plane rational curves of degree $d$ through $3 d-1$ points in $\mathbb{P}^{2}$. Kontsevich's recursion formula [14] reads

$$
n_{d}=\sum_{k=1}^{d-1} n_{k} n_{d-k}\left[k^{2}(d-k)^{2}\left(\begin{array}{l}
3 d-4 \\
3 k-2
\end{array}\right)-k^{3}(d-k)\left(\begin{array}{c}
3 d-4 \\
3 k-1
\end{array}\right)\right], \quad n_{1}=1
$$


The result is $n_{1}=1, n_{2}=1, n_{3}=12$, etc, ie. there is 1 line through 2 points of the plane, 1 conic through 5 points of the plane, 12 cubics through 8 points of the plane, etc.

We can similarly draw tables of $n_{d} \bmod k$ for any integer $k$. The results (in the same convention as before) are:

$* k=2$ : both rows vanish (except first two values), ie. all $n_{d}$ are even.

$* k=2^{l}$ : all rows are 0 , ie $n_{d} \equiv 0 \bmod 2^{l}$ for $n>l+1$.

* $k=3: n_{3 d} \equiv 0 \bmod 3, n_{3 d+2} \equiv 1 \bmod 3, n_{3 d+1} \equiv$ alternating 1 or $2 \bmod 3$ because $n_{6 d+2} \equiv 4 \bmod 6$.

* $k=5: n_{d} \equiv 0 \bmod 5$, for $d>8$. Idem for $k=25(d>23)$

Because of this big symmetry for low primes, most non-primes will yield constant or regular rows (ie rows repeating when shifting horizontally). The only non-obvious case is $k=26$, where there is a shift by 8 (because $k=13$ shifts by 16) and rows 4,6 alternate with 0 .

Further, we only found three primes with regular features:

* $k=7$ : all rows are regular (repeat when shifted horizontally by 4), rows 5 and 7 are 0 .

$* k=13$ : idem, shift by 16 , no 0 row.

$* k=19$ : idem, shift by 12 , no 0 row.

$* k=5,11,17,23,29$ : these primes give almost- 0 rows (ie. $n_{d} \equiv 0$ except for a finite number of $d$ ).

We have not attempted to prove these observations.

\subsubsection{Asymptotics}

We now turn to the asymptotics of the sequence $n_{d}$ for $d \rightarrow \infty$. Di Francesco and Itzykson proved [6. Proposition 3] that

$$
\frac{n_{d}}{(3 d-1) !}=\frac{A^{d}}{d^{7 / 2}}\left(B+\mathrm{O}\left(\frac{1}{d}\right)\right)
$$

as $d$ tends to infinity, and found the approximate values $A \approx 0.138$ and $B \approx 6.1$ for the constants $A$ and $B$. Assuming a full asymptotic expansion

$$
\frac{n_{d}}{(3 d-1) !} \sim \frac{A^{d}}{d^{7 / 2}}\left(B_{0}+\frac{B_{1}}{d}+\frac{B_{2}}{d^{2}}+\cdots\right),
$$

and applying variant (II) of the $\operatorname{asymp}_{k}$ trick to $\log \left(n_{d} /(3 d-1)\right.$ !), we obtain the much more accurate approximations

$$
\begin{aligned}
A & \approx 0.138009346634518656829562628891755541716014121072, \\
B_{0} & \approx 6.0358078488159024106383768720948935,
\end{aligned}
$$

as well as the further values $B_{1} \approx-2.2352424409362074, B_{2} \approx 0.054313787925$. Unfortunately, we are not able to recognize any of these apparently irrational numbers, e.g., PARI does not see in $\log A$ and $\log B_{0}$ a linear combination of simple numbers like 1 , $\log 2, \log 3, \log \pi, \pi$ and $\pi^{2}$. 


\subsection{Numbers of rational curves on the quintic threefold}

The other sequence we now introduce for the purpose of comparison is $q_{d}$, that of holomorphic rational curves of degree $d$ embedded in the quintic Calabi-Yau threefold. These are the 'instanton numbers' of Candelas et al [5]. They are defined by the following line:

$$
5+\sum_{n \geq 1} q_{n} n^{3} \frac{q^{n}}{1-q^{n}}=\left(\frac{q}{x} \frac{d x}{d q}\right)^{3} \frac{5}{\left(1-5^{5} x\right) y_{0}(x)^{2}}=5+2875 q+4876875 q^{2}+\ldots
$$

where $q(x)=x e^{\tilde{y}_{1} / y_{0}}=x+770 x^{2}+\ldots$ is the "mirror map" and its inverse is $x(q)=$ $q-770 q^{2}+\ldots$ The functions $y_{0}$ and $\tilde{y}_{1}$ are solutions of a Picard-Fuchs differential equation and are given by

$$
y_{0}(x):=\sum_{n \geq 0} \frac{(5 n) !}{n !^{5}} x^{n} \quad \text { and } \quad \tilde{y}_{1}(x)=\sum_{n \geq 0}\left(\frac{(5 n) !}{n !^{5}} 5 \sum_{j=n+1}^{5 n} \frac{1}{j}\right) x^{n} .
$$

When computing the numbers $q_{d}$, the longest step is without doubt the inversion of the series $q(x)$. The first few values are $q_{1}=2875, q_{2}=609250$, etc.

We can again draw tables of $q_{d} \bmod k$ for any integer $k$. The main results (in the same convention as before) are:

* $k=2$ : second row is 0 , ie. $q_{2 d}$ are even.

$* k=4,8,16$ : last row is 0 , ie. $q_{2^{l} d} \equiv 0 \bmod 2^{l}(l \leq 4)$

$* k=8,16$ : rows $4,8,12, \ldots$ are also 0 , ie. $q_{2^{l}} d+4 m \equiv 0 \bmod 2^{l}(l \leq 4)$

$* k=32$ : no 0 rows anymore!

$* k=5$ : all rows are 0 , idem at $k=25$.

* $k=20$ : row 4,8,12,16,20 are 0 , because both are $0 \bmod 4$ and $\bmod 5$.

These congruences are much less impressive than in the previous cases, due to the more complicated origin of the instantons. It is not even mathematically understood why these are integers and what exactly they count. Again we have not attempted to prove the congruences.

\subsubsection{Asymptotics}

In this case the asymptotic behaviour is much more tricky than in the previous two examples. The growth is indeed exponential, but $\log q_{d}$ has more than a simple logarithmic term and monomial terms. Indeed, subtracting the logarithmic term gives us a sequence on which the $\operatorname{asymp}_{k}$ trick works badly - as if other logarithmic terms were hiding. In fact, finding out the coefficient of the first log term is already quite tough, and differentiating (to get rid of log-terms) does not yield anything with only monomials. The second author's attempts to deal with the asymptotics of $\log q_{d}$ through the asymp ${ }_{k}$ trick can be found in [11].

\section{Conclusion}

Though the congruences satisfied by the sequence $v_{n}$ of lines in $\mathbb{P}^{n}$ are numerous and very interesting, the exponential asymptotic growth $v_{n} \sim n^{2 n}$ rules out that the $v_{n}$ are 
Fourier coefficients of any modular form on a subgroup of $S L(2, \mathbb{Z})$ (whose coefficients typically grow like $n^{2 k-1}$ for weight $2 k$ ). It is quite gratifying to see that the asymptotic expansion in (10) can be written out with as many exact terms as one wishes, since the coefficients are rational.

Another sequence - that of numbers $n_{d}$ of degree $d$ curves through $3 d-1$ points of the plane - has very similar behaviour, in terms of congruences as well as asymptotics (though the latter's coefficients will be irrational and not recognized). A last sequence - that of numbers $q_{d}$ of degree $d$ rational curves on the quintic Calabi-Yau threefold - is much less enticing; its congruences are rather limited and its asymptotics are awkward: not simply one or two log-terms followed by mere monomial terms, but certainly $\log (\log )$ terms or infinitely many log terms.

It would be interesting to study more of typical sequences from enumerative geometry and see if there is an underlying pattern. Also, the question of how many of those sequences satisfy a recurrence relation is still open. Among the three sequences that we discussed, only that of $n_{d}$ (plane rational curves) obeys a known recurrence.

\section{Acknowledgments}

The first author, who initiated this paper, is deeply grateful for Don Zagier's help throughout all the stages of this project. Not only did Don simplify matters considerably with his formula (3), but he also equipped him with the asymp $k$ trick. He is thankful for fruitful discussions with Robert Osburn. The second author likes to thank Daniel Berend, Paolo Dominici and Yossi Moshe for interesting e-mail correspondence and Carl Pomerance for providing us with Lemma 15. He especially likes to thank Don Zagier for his help in improving the exposition of the paper. Dmitry Kerner and Masha Vlasenko we thank for several helpful discussions. Several inaccuracies in an earlier version were detected by Alexander Blessing during a two week practicum he did with the second author.

This project was made possible thanks to the support of the MPIM in Bonn.

\section{APPENDIX \\ by Don Zagier \\ Exact and asymptotic formulas for $v_{n}$}

In this appendix we prove the alternative definition (3) and the asymptotic formulae (4) and (10) for the numbers $v_{n}$ defined in (2).

\section{Exact formulas}

Proposition 1 Let $G(x, y)$ be a homogeneous polynomial of degree $2 n$ in two variables and $P(x)$ a monic polynomial of degree $n+1$ with distinct roots. Then the expression

$$
\sum_{\substack{\alpha, \beta \in \mathbb{C} \\ P(\alpha)=P(\beta)=0}} \frac{G(\alpha, \beta)}{P^{\prime}(\alpha) P^{\prime}(\beta)}
$$

is independent of $P$ and equals the coefficient of $x^{n} y^{n}$ in $G(x, y)$. 
Proof. By linearity it is enough to consider monomials $G(x, y)=x^{r} y^{s}, r+s=2 n$. Then the expression (12) factors as $\left(\sum_{P(\alpha)=0} \frac{\alpha^{r}}{P^{\prime}(\alpha)}\right)\left(\sum_{P(\beta)=0} \frac{\beta^{s}}{P^{\prime}(\beta)}\right)$. But by the residue theorem we have

$$
\sum_{P(\alpha)=0} \frac{\alpha^{r}}{P^{\prime}(\alpha)}=\sum_{\alpha \in \mathbb{C}} \operatorname{Res}_{x=\alpha}\left(\frac{x^{r} d x}{P(x)}\right)=-\operatorname{Res}_{x=\infty}\left(\frac{x^{r} d x}{P(x)}\right),
$$

and this equals 0 if $0 \leq r<n$ and 1 if $r=n$ since $P$ is monic of degree $n+1$. The proposition follows.

Remark. The same proof shows that if $G$ is homogeneous of degree $m+n$ and $P$ and $Q$ are two monic polynomials of degrees $m+1$ and $n+1$ with distinct roots, then

$$
\sum_{P(\alpha)=Q(\beta)=0} \frac{G(\alpha, \beta)}{P^{\prime}(\alpha) Q^{\prime}(\beta)}
$$

is independent of $P$ and $Q$ and is equal to the coefficient of $x^{m} y^{n}$ in $G(x, y)$. Yet more generally, and still with the same proof, if $G$ is a homogeneous polynomial of degree $n_{1}+\cdots+n_{k}$ in $k$ variables and $P_{1}, \ldots, P_{k}$ monic polynomials of degree $n_{1}+1, \ldots, n_{k}+1$ with no multiple roots, then

$$
\sum_{P_{1}\left(\alpha_{1}\right)=\ldots=P_{k}\left(\alpha_{k}\right)=0} \frac{G\left(\alpha_{1}, \ldots, \alpha_{k}\right)}{P_{1}^{\prime}\left(\alpha_{1}\right) \cdots P_{k}^{\prime}\left(\alpha_{k}\right)}
$$

is independent of all the $P_{i}$ and is equal to the coefficient of $x_{1}^{n_{1}} \cdots x_{k}^{n_{k}}$ in $G\left(x_{1}, \ldots, x_{k}\right)$. In fact $G$ need not even be homogeneous, but can be any polynomial in $k$ variables of degree $\leq n_{1}+\cdots+n_{k}$.

Corollary 2 Let $F(x, y)$ be a symmetric homogeneous polynomial of degree $2 n-2$ in two variables and $w_{0}, \ldots, w_{n}$ distinct complex numbers. Then the expression

$$
\sum_{0 \leq i<j \leq n} \frac{F\left(w_{i}, w_{j}\right)}{\prod_{\substack{0 \leq k \leq n \\ k \neq i, j}}\left(w_{i}-w_{k}\right)\left(w_{j}-w_{k}\right)}
$$

is independent of $w_{0}, \ldots, w_{n}$ and equals the coefficient of $x^{n-1}$ in $(1-x) F(x, 1)$.

Proof. This follows after a short calculation if we apply the proposition to $G(x, y)=$ $(x-y)^{2} F(x, y), P(x)=\prod_{i=0}^{n}\left(x-w_{i}\right)$.

Corollary 2 immediately implies that the right hand side of equation (2) is independent of the (distinct) complex variables $w_{0}, \ldots, w_{n}$ and that (2) is equivalent to (3). The computational advantage is huge: formula (2) is very slow to compute, even for moderately large $n$, whereas (3) can be implemented in PARI in one line as

$$
v(n)=\operatorname{coeff}(\operatorname{prod}(j=0,2 * n-3,2 * n-3-j+j * x, 1-x), n-1)
$$

and takes $<2$ seconds to compute $v_{n}$ up to $n=100$ and 46 seconds up to $n=224$. 
We can rewrite (3) in several other forms by using residue calculus. Setting $D=2 n-3$ and making the substitution $x=1-D / z$, we find

$$
\begin{aligned}
v_{n} & =\operatorname{Res}_{x=0}\left((1-x) \prod_{j=0}^{2 n-3}(2 n-3-j+j x) \frac{d x}{x^{n}}\right) \\
& =D^{2 n} \operatorname{Res}_{z=D}\left(\frac{\prod_{j=0}^{D}(z-j)}{z^{n+1}(z-D)^{n}} d z\right) .
\end{aligned}
$$

Since the residue of the integrand at infinity is zero, we can also write this as

$$
v_{n}=-D^{2 n} \operatorname{Res}_{z=0}\left(\frac{\prod_{j=0}^{D}(z-j)}{z^{n+1}(z-D)^{n}} d z\right),
$$

while simply making the substitution $z \mapsto D-z$ in (14) gives the similar expression

$$
v_{n}=D^{2 n} \operatorname{Res}_{z=0}\left(\frac{\prod_{j=0}^{D}(z-j)}{z^{n}(z-D)^{n+1}} d z\right)
$$

and adding these two last expressions gives yet a third form:

$$
v_{n}=\frac{1}{2} D^{2 n+1} \operatorname{Res}_{z=0}\left(\frac{\prod_{j=0}^{D}(z-j)}{z^{n+1}(z-D)^{n+1}} d z\right) .
$$

Each of the formulae (15)-(17) expresses $v_{n}$ as the constant term at $z=0$ of the Laurent expansion of a rational function, e.g. (15) says

$$
v_{n}=(-1)^{n} D^{2 n} \cdot \text { coefficient of } z^{n-1} \text { in } \frac{(1-z)(2-z) \cdots(D-1-z)}{(D-z)^{n-1}} \text { as } z \rightarrow 0 .
$$

Substituting $z=D u$, we can write this as

$$
v_{n}=(-1)^{n} D^{2} \cdot \text { coefficient of } u^{n-1} \text { in } \frac{(1-D u)(2-D u) \cdots(D-1-D u)}{(1-u)^{n-1}} \text { as } u \rightarrow 0,
$$

from which we see again that $D^{2} \mid v_{n}$ (Lemma 10. By expanding $(D-z)^{1-n}$ by the binomial theorem, we also obtain closed formulae for $v_{n}$; for instance (18) gives:

$$
v_{n}=\sum_{m=0}^{n-1}(-1)^{n-1-m}\left(\begin{array}{c}
2 n-2-m \\
n-1
\end{array}\right) D^{m+1}\left[\begin{array}{l}
D \\
m
\end{array}\right]
$$

where $\left[\begin{array}{c}D \\ m\end{array}\right]$, the coefficient of $z^{m}$ in $z(z+1) \cdots(z+D-1)$, is a Stirling number of the first kind.

\section{Asymptotics}

To obtain the asymptotic expansion of $v_{n}$, we write the residue in (13) as $\frac{1}{2 \pi i} \int_{|x|=1}$ and we make the substitution $x=(1+i t) /(1-i t)$ to obtain, after a short calculation,

$$
v_{n}=\frac{2}{\pi} \int_{-\infty}^{\infty} \prod_{r=1,3, \ldots, D}\left(\frac{D^{2}+r^{2} t^{2}}{1+t^{2}}\right) \frac{t^{2} d t}{\left(1+t^{2}\right)^{2}}=\frac{2}{\pi} D^{D+1} \int_{-\infty}^{\infty} \phi_{D}(t) \frac{t^{2} d t}{\left(1+t^{2}\right)^{2}}
$$


where $\phi_{D}(t)$ denotes the rational function

$$
\phi_{D}(t)=\prod_{r=1,3, \ldots, D} \frac{1+r^{2} D^{-2} t^{2}}{1+t^{2}}
$$

It is easy to see that $\phi_{D}(0)=1$ and $\phi_{D}(t) \leq e^{-c D t^{2}}$ for some absolute constant $c>0$ (a much more precise formula will be given in a moment), so the main contribution to the integral comes from small $t$. For $t$ small and $D$ large we have (uniformly in both variables)

$$
\begin{aligned}
& \log \phi_{D}(t)=\sum_{j=1}^{\infty} \frac{(-1)^{j-1}}{j}\left[\sum_{r=1,3, \ldots, D}\left(\frac{r^{2 j}}{D^{2 j}}-1\right)\right] t^{2 j} \\
& =\left(-\frac{D}{3}+\frac{1}{3 D}\right) t^{2}+\left(\frac{D}{5}-\frac{1}{3 D}+\frac{2}{15 D^{3}}\right) t^{4}+\left(-\frac{D}{7}+\frac{1}{3 D}-\frac{4}{9 D^{3}}+O\left(\frac{1}{D^{5}}\right)\right) t^{6} \\
& +\left(\frac{D}{9}-\frac{1}{3 D}+\frac{14}{15 D^{3}}+O\left(\frac{1}{D^{5}}\right)\right) t^{8}+\left(-\frac{D}{11}+\frac{1}{3 D}+O\left(\frac{1}{D^{3}}\right)\right) t^{10} \\
& +\left(\frac{D}{13}-\frac{1}{3 D}+O\left(\frac{1}{D^{3}}\right)\right) t^{12}+\left(-\frac{D}{15}+O\left(\frac{1}{D}\right)\right) t^{14}+\left(\frac{D}{17}+O\left(\frac{1}{D}\right)\right) t^{16}+O\left(D t^{18}\right),
\end{aligned}
$$

and hence

$$
\begin{aligned}
& \frac{x^{2}}{\left(1+x^{2} / D\right)^{2}} \phi_{D}\left(\frac{x}{\sqrt{D}}\right) \\
& =e^{-x^{2} / 3}\left[x^{2}+\left(\frac{x^{6}}{5}-2 x^{4}\right) D^{-1}+\left(\frac{x^{10}}{50}-\frac{19 x^{8}}{35}+3 x^{6}+\frac{x^{4}}{3}\right) D^{-2}\right. \\
& +\left(\frac{x^{14}}{750}-\frac{12 x^{12}}{175}+\frac{314 x^{10}}{315}-\frac{59 x^{8}}{15}-x^{6}\right) D^{-3}+\cdots \\
& \left.+\left(\frac{x^{30}}{393750000}-\frac{11 x^{28}}{19687500}+\cdots+\frac{355 x^{10}}{162}+\frac{2 x^{8}}{45}\right) D^{-7}+O\left(D^{-8}\right)\right] .
\end{aligned}
$$

Substituting this expansion (with the 34 omitted terms included) into equation (21) with $t$ replaced by $x / \sqrt{D}$ and using the standard evaluation

$$
\int_{-\infty}^{\infty} e^{-x^{2} / 3} x^{2 n} d x=\frac{(2 n) !}{n !}\left(\frac{3}{4}\right)^{n} \sqrt{3 \pi}
$$

we obtain

$$
\begin{aligned}
v_{n} & =\sqrt{\frac{27}{\pi}} D^{D-1 / 2}\left(1-\frac{9}{4} D^{-1}+\frac{969}{160} D^{-2}-\frac{61479}{3200} D^{-3}+\frac{25225773}{358400} D^{-4}\right. \\
& \left.-\frac{10092025737}{35840000} D^{-5}+\frac{2271842858513}{2007040000} D^{-6}-\frac{4442983688169}{1146880000} D^{-7}+O\left(D^{-8}\right)\right) .
\end{aligned}
$$

This asymptotic formula can of course be written in many other ways, e.g.:

$$
v_{n}=\sqrt{\frac{27}{\pi}}(2 n-3)^{2 n-7 / 2}\left(1-\frac{9}{8 n}-\frac{111}{640 n^{2}}-\frac{9999}{25600 n^{3}}+\frac{87261}{5734400 n^{4}}-\cdots\right)
$$

or

$$
v_{n}=e^{-3} \sqrt{\frac{27}{\pi}}(2 n)^{2 n-7 / 2}\left(1+\frac{15}{8 n}+\frac{1689}{640 n^{2}}+\frac{79281}{25600 n^{3}}+\frac{19691853}{5734400 n^{4}}+\cdots\right)
$$


or

$$
\log \frac{v_{n}}{(2 n) !}=2 n-4 \log n+C+\frac{11}{6 n}+\frac{141}{160 n^{2}}+\frac{9973}{28800 n^{3}}+\frac{59673}{179200 n^{4}}+\cdots
$$

with $C=-3-\log \pi-\frac{3}{2} \log \frac{8}{3}$. Of course, more terms could be obtained in any of these expansions if desired.

\section{References}

[1] F.L. Bauer, For all primes greater than $3,\left(\begin{array}{c}2 p-1 \\ p-1\end{array}\right) \equiv 1\left(\bmod p^{3}\right)$ holds, Math. Intelligencer 10 (1988), no. 3, 42.

[2] D. Berend and J.E. Harmse, On some arithmetical properties of middle binomial coefficients, Acta Arith. 84 (1998), 31-41.

[3] F. Beukers, Irrationality proofs using modular forms, Astérisque No. 147-148 (1987), $271-283$.

[4] T. Cai, A congruence involving the quotients of Euler and its applications. I, Acta Arith. 103 (2002), 313-320.

[5] P. Candelas, J.X. de la Ossa, P. Green, and L. Parkes, A Pair of Calabi-Yau Manifolds as an Exactly Soluble Superconformal Field Theory, Nucl. Phys. B359 (1991), 21-74, reprinted in Essays on Mirror Manifolds (S.T. Yau, ed.) Hong Kong, 1992.

[6] P. Di Francesco and C. Itzykson, Quantum intersection rings. The moduli space of curves (Texel Island, 1994), 81-148, Progr. Math., 129, Birkhäuser Boston, Boston, MA, 1995.

[7] P. Dominici, Sequence A027363, On-Line Encyclopedia of Integer Sequences, http://www.research.att.com/ njas/sequences/

[8] W. Fulton, Intersection Theory, Springer, New York, 1984.

[9] A. Granville, Arithmetic properties of binomial coefficients. I. Binomial coefficients modulo prime powers, Organic mathematics (Burnaby, BC, 1995), 253-276, CMS Conf. Proc., 20, Amer. Math. Soc., Providence, RI, 1997.

[10] D. Grünberg, Integrality of open instanton numbers, J. Geom. Phys. 52 (2004), 284-297, hep-th/0305057.

[11] D. Grünberg, Asymptotic growth of the sequence of instantons on the quintic threefold, unpublished manuscript, 2005.

[12] G.H. Hardy and E.M. Wright, An introduction to the theory of numbers. Fifth edition. The Clarendon Press, Oxford University Press, New York, 1979.

[13] S. Katz and S.A. Stromme, Schubert - a Maple package for intersection theory and enumerative geometry, (1992) http://www.mi.uib.no/〜stromme/schubert/

[14] M. Kontsevich and Y. Manin, Gromov-Witten classes, quantum cohomology, and enumerative geometry, Comm. Math. Phys. 164 (1994), 525-562, hep-th/9402147.

[15] L. Manivel, Symmetric functions, Schubert polynomials and degeneracy loci, SMF/AMS Texts and Monographs 6, American Mathematical Society, Providence, RI, 2001. 
[16] Y. Moshe, The density of 0's in recurrence double sequences, J. Number Theory 103 (2003), 109-121.

[17] Y. Moshe, The distribution of elements in automatic double sequences, Discrete Math. 297 (2005), 91-103.

[18] J. Stienstra and F. Beukers, On the Picard-Fuchs equation and the formal Brauer group of certain elliptic K3-surfaces, Math. Ann. 271 (1985), 269-304.

[19] B.L. van der Waerden, Zur algebraischen Geometrie. II. Die geraden Linien auf den Hyperflächen des $\mathbb{P}_{n}$. Math. Ann. 108 (1933), 253-259.

[20] B.L. van der Waerden, Zur algebraischen Geometrie. Selected papers, Springer-Verlag, Berlin, 1983.

[21] D. Zagier, Vassiliev invariants and a strange identity related to the Dedekind eta-function, Topology 40 (2001), 945-960.

D. Grünberg, 49 rue Fondary, 75015 Paris, e-mail: grunberg@mccme.ru

P. Moree, Max-Planck-Institut für Mathematik, Vivatsgasse 7, D-53111 Bonn, Germany.

e-mail: moree@mpim-bonn.mpg.de

D. Zagier, Max-Planck-Institut für Mathematik, Vivatsgasse 7, D-53111 Bonn, Germany.

e-mail: don@mpim-bonn.mpg.de 